\title{
Networks of manufacturers and retailers
}

A. Mauleon, J. Sempere-Monerris and V. Vannetelbosch

Discussion Paper 2005-36

\section{Département des Sciences Économiques de l'Université catholique de Louvain}




\title{
Networks of Manufacturers and Retailers*
}

\author{
Ana Mauleon ${ }^{1}, J_{0 s e}$ Sempere-Monerris ${ }^{2}$ and Vincent J. Vannetelbosch ${ }^{3}$
}

${ }^{1}$ FNRS, CEREC, Facultés Universitaires Saint-Louis and CORE, Boulevard du Jardin Botanique 43, 1000 Brussels, Belgium (e-mail: mauleon@fusl.ac.be)

${ }^{2}$ Department of Economic Analysis, University of Valencia, Campus dels Tarongers, Avd. dels Tarongers s/n, 46022 Valencia, Spain (e-mail: Jose.J.Sempere@uv.es)

3 FNRS and CORE, Université catholique de Louvain, Voie du Roman Pays 34, 1348 Louvain-la-Neuve, Belgium (e-mail: vannetelbosch@core.ucl.ac.be)

\begin{abstract}
We study the endogenous formation of networks between manufacturers of differentiated goods and multi-product retailers who interact in a successive duopoly. Joint consent is needed to establish and/or maintain a costly link between a manufacturer and a retailer. We find that only three distribution networks are stable for particular values of the degree of product differentiation and link costs: (i) the non-exclusive distribution \& non-exclusive dealing network in which both retailers distribute both products is stable for intermediate degree of product differentiation and small link costs; (ii) the exclusive distribution \& exclusive dealing network in which each retailer distributes a different product is stable for low degrees of product differentiation; (iii) the mixed distribution network in which one retailer distributes both products while the other retailer sells only one is stable for high degrees of product differentiation and large link costs. We show that the distribution networks that maximize social welfare are not necessarily stable. Thus, a conflict between stability and social welfare is likely to occur, even more if the degree of product differentiation is either low or high.
\end{abstract}

JEL Classification: C70, L13, L20, J50, J52

Key words: Networks, Retailers, Manufacturers.

\footnotetext{
*Ana Mauleon and Vincent Vannetelbosch are Research Associates of the National Fund for Scientific Research (FNRS), Belgium. Vincent Vannetelbosch is Associate Fellow of CEREC, Facultés Universitaires Saint-Louis.
} 


\section{Introduction}

In various industries, such as e.g. automobiles, clothing, electronics, pharmaceuticals, and food, manufacturers develop networks of exchange both with input suppliers and retailers or wholesalers. Moreover, in the last few decades the importance of spot exchange in input or output procurement has decreased in favor of other methods such as manufacturer-supplier long-term contracting and manufacturer-retailer exchange networks. For automobiles, Nishiguchi (1994) has presented wide ranging evidence on the ways in which the Japanese industrial model has evolved from the traditional bargainingoriented manufacturer-supplier relationship to the current problem-solving-driven strategic industrial outsourcing. Firms rely more and more on a subset of suppliers with whom they maintain closed business ties. The number of direct suppliers to Japanese car manufacturers in 1988 was roughly one half of what it was for American or European manufacturers, for similar volumes of production. The manufacturer-retailer relationship for the European motor vehicle industry has also evolved over the last years. Until October 2002, only one type of distribution (a system of exclusive territories and selectivity) was permitted. The European Commission was not satisfied with the unexplained differences in prices across European countries and this motivated a legal change. The new regulation recently issued $^{1}$ is seeking for a change in the car distribution industry. As Commissioner Monti said: "The new rules that will become effective as of 1 October 2003 open the way to new distribution techniques, such as Internet sales and multi-branding - introducing more competition between different retail channels". ${ }^{2}$ Then it is expected that multi-branding dealers will appear and coexist with exclusive ones.

The literature on network formation has focused on the upstream part of the vertical chain, neglecting the analysis of the downstream part where manufacturers and retailers enter in long-term relationships. ${ }^{3}$ Kranton and Minehart (2000a) have examined the emergence of buyer-seller networks when sellers have an outsourcing motivation in order to see whether networks of buyers and sellers can perform better than vertically integrated markets or spot exchange markets. Manufacturers can decide to build a dedicated

\footnotetext{
${ }^{1}$ Regulation 1400/2002 on the application of Article 81(3) of the Treaty to categories of vertical agreements and concerted practices in the motor vehicle sector.

${ }^{2}$ Extracted from "New rules for car sales and servicing" (September 2003) and European Commissioner Monti's speech "The new legal framework for car distribution" (February 2003). See http://www.europa.eu.int/comm/competition/ for more details.

${ }^{3}$ The data in Betancourt (2004) suggests that there has been substantial forward vertical integration by manufacturers in the form of internalizing the wholesale function by selling directly to retailers. This process is most pronounced in the durable sectors: automobiles and other motor vehicles, 95.5 percent of sales; electronics, 70.9 percent of sales; toys and hobby goods, 95.6 percent of sales (US retail sector in 1987 ).
} 
asset to produce their own inputs or, alternatively, they can invest in links to external sellers from which they will buy specialized inputs. They have established a connection between industrial structure and uncertainty in demand: outsourcing networks appear to be more efficient than vertically integrated structures when uncertainty in demand is substantial. Kranton and Minehart (2001) have focused on when the noncooperative formation of buyer-seller networks leads to the formation of efficient networks, while Kranton and Minehart (2000b) have examine the competitive equilibrium prices in buyer-seller networks. Wang and Watts (2003) have analyzed the formation of buyer-seller links when sellers can produce products of different quality. ${ }^{4}$ In this paper, we are first to examine the emergence of manufacturer-retailer networks when both manufacturers and retailers decide the bilateral links they want to establish among them.

The literature on distribution systems initially addressed two questions: (i) whether manufacturers would prefer having a single common retailer rather than separate exclusive retailers; ${ }^{5}$ and (ii) whether a manufacturer's brand is excluded from the market by use of exclusive contracts. ${ }^{6}$ A series of papers have studied the distribution systems that arise when there is market power at both the manufacturing and retailing levels. ${ }^{7}$ In particular, for the successive duopoly case, Chang(1992) and Dobson and Waterson (1997) have analyzed the distribution systems that arise by the joint maximization of the manufacturerretailer pair profits, allowing for side-payments if an exclusive contract is signed. Chang (1992) has found that manufacturer-retailer pairs always choose exclusive dealing. Once manufacturers and retailers are differentiated, Dobson and Waterson (1997) have shown

\footnotetext{
${ }^{4}$ There is a vast literature devoted to analyze the important role played by network structures in determining the outcome of many other economic situations. For example, Hendricks, Piccione and Tang (1997) have shown that the structure of airline connections influences competition. Belleflamme and Bloch (2004), Goyal and Moraga (2001) and Goyal and Joshi (2003) have studied the formation of research and development networks and collusive alliances among corporations. Calvó-Armengol (2004) and CalvóArmengol and Jackson (2004) have examined the role played by personal contacts in obtaining information about job opportunities.

${ }^{5}$ An exclusive dealing agreement is a restriction of the retailer's behavior under which the retailer agrees not to buy from any other manufacturer. Similarly, an exclusive distribution agreement is a manufacturer's behavior restriction under which the manufacturer agrees not to sell to any other retailer. Lin (1990) and O'Brien and Shaffer (1993) have shown that exclusive dealership rather than common dealership is chosen to dampen competition between the manufacturers.

${ }^{6}$ In a setting with two manufacturers and only one retailer, O’Brien and Shaffer (1997) and Bernheim and Whinston (1998) have shown that vertical foreclosure is not an equilibrium. We refer to Rey and Tirole (2003) for a complete survey on vertical foreclosure.

${ }^{7}$ The possibility of a manufacturer hiring more than one retailer has been considered out of the successive duopoly structure. See Rey and Stiglitz (1995) for the case where manufacturers can hire several retailers in a perfect competition setting, and Besanko and Perry (1994) for the case of spatially differentiated retailers whose number is endogenously determined by free entry.
} 
that the manufacturer-retailer pairs prefer non-exclusive dealing contracts when products and retailers are sufficiently differentiated. Moner-Colonques, Sempere-Monerris and Urbano (2004) have analyzed a successive duopoly where two manufacturers with asymmetric and differentiated brands choose strategically how many undifferentiated retailers to employ. When product differentiation is strong and brand asymmetry is moderate, both manufacturers distribute through both retailers. However, when both product differentiation and brand asymmetry are weak, exclusive dealing through a single retailer is used. There are also asymmetric equilibria in which one manufacturer distributes through both retailers but the other manufacturer distributes through one retailer. These equilibria can arise when both product differentiation and brand asymmetry are strong. Finally, Mycielski, Riyanto and Wuyts (2000) have studied manufacturers' choice of two types of vertical arrangement with retailers; exclusive dealing and exclusive territory. When products are less substitutable, in other words, the interbrand rivalry is weak, manufacturers prefer to sell brands to a large number of competitive retailers. When the interbrand rivalry is strong, exclusive territory with exclusive dealing is adopted by manufacturers.

In this paper we address the following questions:

(i) What are the incentives of manufacturers and retailers to link and what is the architecture of "stable" networks of distribution when both manufacturers and retailers decide the bilateral links they want to establish among them?

(ii) Are individual incentives to link adequate from a social welfare point of view?

In order to answer these questions we develop a three-stage game in a successive duopoly. Each manufacturer produces a differentiated product (brand) which is sold to retailers at a constant per unit price and retailers can be multiproduct sellers. In the first stage, the two manufacturers and the two retailers decide about bilateral relationships (or links) they want to establish among them. A link between a manufacturer and a retailer is necessary in order to sell the manufacturer's brand to consumers. The cost of a link is shared equally between the manufacturer and the retailer. ${ }^{8}$ The collection of pairwise links between manufacturers and retailers defines a distribution network. In the second stage, both manufacturers choose simultaneously the terms of trade of their good to retailers (transfer prices). In the third stage, both retailers compete by setting simultaneously the quantity of each brand they are going to market.

\footnotetext{
${ }^{8}$ In the motor vehicle industry the "just-in-time" philosophy has been present for decades. It requires the coordination and collaboration across organizations and throughout the supply chain. This means that there should be a permanent relationship among them and this is costly. Also, it is becoming more common to find a supply channel management approach in different industries where independent members of the supply chain coordinate in the management of such a chain.
} 
A simple way to analyze the networks that one might expect to emerge in the long run is to examine a sort of equilibrium requirement that agents not benefit from altering the structure of the network. A weak version of such condition is the pairwise stability notion defined by Jackson and Wolinsky (1996). A network is pairwise stable if no agent benefits from severing one of their links and no other two agents benefit from adding a link between them, with one benefiting strictly and the other at least weakly. While pairwise stability is natural and quite easy to work with, there are some limitations of the concept. First, it is a weak notion in that it only considers deviations on a single link at a time. For instance, it could be that an agent would not benefit from severing any single link but would benefit from severing several links simultaneously, and yet the network would still be pairwise stable. Second, pairwise stability considers only deviations by at most a pair of agents at a time. It might be that some group of agents could all be made better off by some complicated reorganization of their links, which is not accounted for under pairwise stability. A strongly stable network, whose definition is due to Jackson and van den Nouweland (2005), is a network which is stable against changes in links by any coalition of agents. ${ }^{9}$

Stable networks obtained from the joint consent of the agents involved might result in distribution networks that coincide with those resulting from both manufacturers and retailers signing exclusive dealing or exclusive distribution contracts. Thus, this model might be used by the competition authorities to distinguish whether exclusive dealing is agreed by all agents in the market (i.e. not imposed by one kind of agent), whether it is efficient and whether it is socially optimal. Exclusive dealing as many as other nonprice vertical restraints is challenged by competition authorities. The legal treatment of nonprice vertical restraints has not been uniform along the years. In the U.S. vertical restraints were initially considered as not per se illegal, then per se illegal and now a rule of reason is applied. The analytical justification for a rule of reason is the twofold effect of nonprice vertical restraints in general and exclusive dealing in particular. ${ }^{10}$ Vertical restraints have a procompetitive effect when they are used to avoid the double marginalization inefficiency or to reduce the underprovision of services that affect the demand of the good. Vertical restraints have an anticompetitive effect when they are used to reduce or eliminate intrabrand competition (same brand is sold at different outlets), to dampen competition at the upstream levels, or to foreclose market access and prevent entry. The procompetitive effect is more likely to dominate the anticompetitive effect provided interbrand competition

\footnotetext{
${ }^{9}$ Jackson $(2003,2005)$ provides surveys of models of network formation.

${ }^{10}$ Caballero-Sanz and Rey (1996) and Dobson and Waterson (1997) have provided a detailed analysis of the economic evaluation of vertical restraints and the implications for competition policy.
} 
is sufficiently strong. ${ }^{11}$

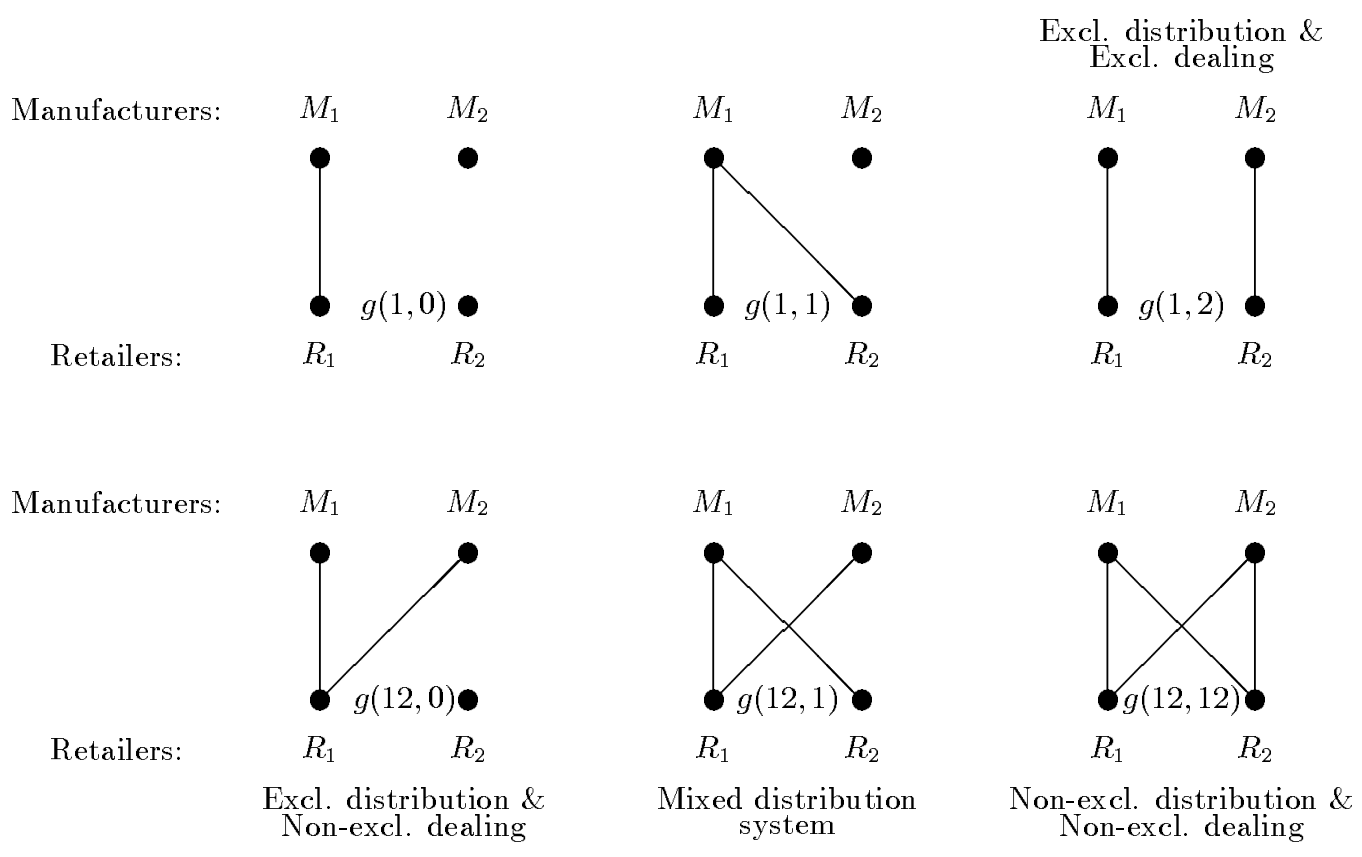

Figure 1: The six qualitatively different distribution networks.

In a successive duopoly, there are fifteen possible network architectures. But given the symmetry of products and retailers, there are only six qualitatively different distribution networks which are depicted in Figure 1. Depending on the distribution network, two kinds of competition may be eventually at place: interbrand competition and intrabrand competition. Therefore, the distribution network that will emerge is the result of the interplay of two effects: first, the one associated to the cost of implementing a particular network which depends on both the link cost size and the number of links; and a second one which is associated to the combination of inter and intrabrand competition that arises in each particular network. Given that agents act strategically and in their self-interest, the stable distribution network might differ from the one preferred by consumers or the

\footnotetext{
${ }^{11}$ This point has been included in the $\mathrm{EC}$ Guidelines on vertical restraints: "The market position of the supplier and his competitors is of major importance, as the loss of intrabrand competition can be problematic if interbrand competition is limited. The stronger the position of the supplier, the more serious is the loss of intrabrand competition..." (see Official Journal of the European Communities, C 291, 13/10/2000). Recently, the European Commission has exempted for five years certain exclusive distribution agreements between Telenor and Canal + Nordic, under which Telenor will have the exclusive right to distribute Canal + Nordic's premium pay-TV channels in the Nordic region through its satellite television platform Canal Digital. The argument of this exemption was the presence of a second satellite pay-TV distributor in the Nordic region, MTG/Viasat and that consumers would have available two distinct pay-TV brands at competitive prices, i.e. sufficient interbrand competition (see the IP/04/2, January 5 , 2004 and the EC Commission Competition Policy Newsletter, Summer 2004).
} 
one that maximizes social welfare.

We find that only three distribution networks are strongly stable for particular values of the degree of product differentiation and link costs. A first distribution network with four links, referred as non-exclusive distribution \& non-exclusive dealing, in which both retailers distribute both products is strongly stable for intermediate degree of product differentiation and small link costs. In this distribution network, both interbrand and intrabrand competition are present in the market. A second distribution network with two links, referred as exclusive distribution \& exclusive dealing, in which each retailer distributes a different product is strongly stable for low degrees of product differentiation. In this distribution network, no intrabrand competition appears in the market. A third distribution network with three links, referred as mixed distribution system, in which one retailer distributes both products while the other retailer sells only one is strongly stable for high degrees of product differentiation and large link costs. Finally, for some values of the degree of product differentiation and link costs, no distribution network is strongly stable. In particular, when the degree of product differentiation is high enough or intermediate, the non-exclusive distribution \& non-exclusive dealing system will not emerge in the "longrun" while Mycielski, Riyanto and Wuyts (2000) and Moner-Colonques, Sempere-Monerris and Urbano (2004) have shown that it is a "short-run" equilibrium. ${ }^{12}$

We also wonder whether the stable distribution network is efficient, in the sense that it generates the greatest surplus for the agents that integrate the network. We find that the three stable distribution networks can be efficient for particular values of the degree of product differentiation and link costs, but not necessarily for the values under which they are stable. Moreover, the distribution network, referred as exclusive distribution \& nonexclusive dealing, in which two manufacturers distribute their products using a single and identical retailer is never stable but it is efficient for low degrees of product differentiation. Thus, a conflict between stability and efficiency may occur.

Since consumers do not account for link costs, the non-exclusive distribution \& nonexclusive dealing system maximizes consumer surplus. Thus, consumers are better off the highest the level of competition. That is, they are better off in a market with interbrand and intrabrand competition in both products. Social welfare is maximized by either of the four efficient distribution networks depending on the degree of product differentiation and on the link costs. When link costs are small enough, two distribution networks may

\footnotetext{
${ }^{12}$ In Mycielski, Riyanto and Wuyts (2000), the two manufacturers choose simultaneously among combinations of vertical arrangements; exclusive distribution, non-exclusive distribution, exclusive dealing, and non-exclusive dealing. In Moner-Colonques, Sempere-Monerris and Urbano (2004), two manufacturers choose simultaneously whether to employ retailer one, retailer two, both or neither of them. Both papers use the subgame perfect Nash equilibrium to solve the game and assume that links are costless.
} 
maximize social welfare. The non-exclusive distribution \& non-exclusive dealing system maximizes social welfare if the degree of product differentiation is high enough; otherwise, the mixed distribution system maximizes social welfare. When link costs become large, two other distribution networks may maximize welfare. The exclusive distribution \& exclusive dealing system maximizes welfare if the degree of product differentiation is high enough; otherwise, the exclusive distribution \& non-exclusive dealing system maximizes welfare. Thus, a conflict between stability and social welfare is likely to occur, even more if the degree of product differentiation is either low or high. ${ }^{13}$

The paper is organized as follows. The model is presented in Section 2. In Section 3 we analyze the stable distribution networks. In Section 4 we analyze the efficient networks and the networks that maximize consumer surplus and social welfare. Finally, Section 5 concludes.

\section{The model}

We develop a three-stage game to study the formation of networks among manufacturers and retailers in a successive duopoly. To reach consumers manufacturers and retailers should form a product distribution network consisting of different bilateral relationships (or links) between them. In an initial stage, manufacturers and retailers decide the links they want to establish among them. A link between a manufacturer and a retailer is necessary in order to sell the manufacturer's brand to consumers. In the second stage, once the distribution network has been formed, manufacturers decide simultaneously the transfer prices to retailers. Finally, retailers decide simultaneously the quantity of each brand they are going to market.

The two manufacturers $\left(M_{1}\right.$ and $\left.M_{2}\right)$ produce their own branded good under constant returns to scale and incur a common unit cost $c$. The retailers $\left(R_{1}\right.$ and $\left.R_{2}\right)$ are supplied by the manufacturers at a constant unit price, the transfer price. ${ }^{14}$ Let $w_{i}$ denote the transfer

\footnotetext{
${ }^{13}$ Mycielski, Riyanto and Wuyts (2000) have studied the welfare implications of manufacturers' choices of vertical arrangements and its policy implications in a setting where retailers compete à la Bertrand. They have shown that, for a high degree of product differentiation, any policy measure to restrict vertical restraints is unnecessary. However, we get that such policies become necessary once retailers compete à la Cournot. Then, restricting exclusive distribution and exclusive dealing arrangements might have a positive impact on social welfare.

${ }^{14}$ We limit attention to linear contracts. Although the superiority of two-part tariff contracts over linear ones is usually established because with the former manufacturers have two instruments (the transfer price used to give the right incentives to retailers and the fixed fee used to extract all the rent generated by the selling of the good), linear contracts may turn appropriate if there are observability or renegotiation problems (see chapter 4 in Tirole 1988). Linear contracts are used in several industries. Iyer and VillasBoas (2003) have reported that in sectors such as grocery retailing or department stores retailers do not
} 
price set by manufacturer $i$ for supplying brand $i, i=1,2$. We assume that retailers may be multi-product, in the sense that they are allowed to carry both products. We also assume that retailers are not differentiated in the sense that consumers get the same utility for consuming a brand no matter which retailer sells the brand to them. We denote by $N=\left\{M_{1}, M_{2}, R_{1}, R_{2}\right\}$ the set of agents which are connected in a distribution network.

Let $q_{i j}$ be the quantity of brand $i$ that retailer $j$ sells to consumers. In case both retailers distribute brand $i$, let $Q_{i}=q_{i 1}+q_{i 2}$ denote the total amount produced of brand $i$. The retailing costs supported by the retailers are assumed to be zero. Inverse demand functions are given by

$$
\begin{aligned}
& p_{1}=a-Q_{1}-d Q_{2} \\
& p_{2}=a-Q_{2}-d Q_{1}
\end{aligned}
$$

where $a>c$ and $0<d<1$ (own effects on prices are greater than cross effects). So, brands 1 and 2 are imperfect substitutes and parameter $d$ measures the degree of interbrand rivalry, that is, how similar the brands are perceived by consumers. When $d$ approaches 1 brands become closer substitutes (interbrand rivalry increases). Intrabrand rivalry, that is how similar retailers' services are perceived by consumers to be when selling the same brand, is maximal since retailers are not differentiated, they are perfect substitutes. Since retailers can be multi-product sellers, there may be in-store competition, which means interbrand rivalry in a retailer selling the two products.

The distribution network cannot be enforced. We assume that joint consent is needed to establish and/or maintain a link between a manufacturer and a retailer. The cost of maintaining such a distribution link for each agent is denoted by $k \geq 0$. In a distribution network, manufacturers and retailers are the nodes in the graph and links indicate different bilateral relationships between the agents. Then, a distribution network $g$ is simply a list of which pair of manufacturers and retailers are linked to each other. If $M_{1}$ is linked with $R_{1}$ and with $R_{2}$, we write $\left(M_{1}, R_{1}\right) \in g$ and $\left(M_{1}, R_{2}\right) \in g$. In general, if we are considering a pair of agents $i$ and $j$, with $i, j \in N$, then $(i, j) \in g$ indicates that $i$ and $j$ are linked under the network $g$. The network obtained by adding link $(i, j)$ to an existing network $g$ is denoted $g+(i, j)$ and the network obtained by deleting link $(i, j)$ from an existing network $g$ is denoted $g-(i, j)$. Let $G$ be the set of all possible distribution networks.

In what follows, $g(12,0)$ represents the distribution network in which retailer $R_{1}$ is selling brand 1 and brand 2 and retailer $R_{2}$ sells no brand, while $g(1,12)$ represents

seem to pay lump-sum fees to manufacturers. Sass (2005) has described the U.S. beer industry as a three-tier system (brewers, distributors and retailers) where brewers set constant per-unit prices for beer and do not charge distributors explicit franchise fees. Distributors in turn independently set simple linear wholesale prices to retailers. 
the distribution network in which $R_{1}$ is selling brand 1 and $R_{2}$ sells brands 1 and 2; i.e., $g(12,0)=\left\{\left(M_{1}, R_{1}\right),\left(M_{2}, R_{1}\right)\right\}$ and $g(1,12)=\left\{\left(M_{1}, R_{1}\right),\left(M_{1}, R_{2}\right),\left(M_{2}, R_{2}\right)\right\}$. Given the symmetry of products and retailers, there are only six qualitatively different distribution networks out of fifteen. The six distribution networks we are going to analyze are $g(1,0), g(1,1), g(12,0), g(1,2), g(12,1)$ and $g(12,12)$ and are depicted in Figure 1 . The distribution network $g(1,0)$ is symmetric to $g(0,1), g(0,2)$ and $g(2,0) ; g(1,1)$ is symmetric to $g(2,2) ; g(12,0)$ is symmetric to $g(0,12) ; g(1,2)$ is symmetric to $g(2,1)$; and $g(12,1)$ is symmetric to $g(12,2), g(1,12)$ and $g(2,12)$.

Before looking for the stability and efficiency of distribution networks, we derive for each possible network architecture, the equilibrium transfer prices, quantities produced, profits, consumer surplus and aggregate welfare. We denote by $\Pi_{i}(g)$ the profit of $i$ in network $g$. Let $\Phi(g)$ be the sum of the individual payoffs or profits. That is, $\Phi(g)=$ $\Pi_{M_{1}}(g)+\Pi_{M_{2}}(g)+\Pi_{R_{1}}(g)+\Pi_{R_{2}}(g)$. For the sake of the exposition we present here the distribution network $g(12,12)=\left\{\left(M_{1}, R_{1}\right),\left(M_{2}, R_{1}\right),\left(M_{1}, R_{2}\right),\left(M_{2}, R_{2}\right)\right\}$, in which each retailer sells brands 1 and 2 , referred as the non-exclusive distribution \& non-exclusive dealing system.

Agents objective functions in $g(12,12)$ are:

$$
\begin{aligned}
\Pi_{M_{1}}(g(12,12)) & =\left(w_{1}-c\right)\left(q_{11}+q_{12}\right)-2 k \\
\Pi_{M_{2}}(g(12,12)) & =\left(w_{2}-c\right)\left(q_{21}+q_{22}\right)-2 k \\
\Pi_{R_{1}}(g(12,12)) & =\left(p_{1}-w_{1}\right) q_{11}+\left(p_{2}-w_{2}\right) q_{21}-2 k \\
\Pi_{R_{2}}(g(12,12)) & =\left(p_{1}-w_{1}\right) q_{12}+\left(p_{2}-w_{2}\right) q_{22}-2 k
\end{aligned}
$$

In the last stage of the game, links and transfer prices are given. Under Cournot competition the retailers compete by choosing simultaneously the quantity of each brand they are going to market. The unique Nash equilibrium of this stage game is

$$
\begin{aligned}
& q_{11}(g(12,12))=q_{12}(g(12,12))=\frac{a(1-d)-w_{1}+d w_{2}}{3\left(1-d^{2}\right)} \\
& q_{21}(g(12,12))=q_{22}(g(12,12))=\frac{a(1-d)+d w_{1}-w_{2}}{3\left(1-d^{2}\right)} .
\end{aligned}
$$

In the second stage, manufacturers decide simultaneously the transfer prices to retailers. The unique Nash equilibrium of this stage game is

$$
w_{1}(g(12,12))=w_{2}(g(12,12))=w(g(12,12))=a-\frac{(a-c)}{(2-d)} .
$$

Then, one can easily obtain the equilibrium profits: 


$$
\begin{aligned}
& \Pi_{M_{1}}(g(12,12))=\Pi_{M_{2}}(g(12,12))=\frac{2(1-d)(a-c)^{2}}{3(1+d)(2-d)^{2}}-2 k \\
& \Pi_{R_{1}}(g(12,12))=\Pi_{R_{2}}(g(12,12))=\frac{2(a-c)^{2}}{9(1+d)(2-d)^{2}}-2 k .
\end{aligned}
$$

To determine the efficient distribution network, we compute the sum of the individual equilibrium payoffs, $\Phi(g(12,12))$. Then,

$$
\Phi(g(12,12))=\frac{4(4-3 d)(a-c)^{2}}{9(1+d)(2-d)^{2}}-8 k .
$$

Let $C(g(12,12))$ denote the consumer surplus in case $g(12,12)$ is formed. The corresponding consumer surplus for this system of inverse linear demands is given by the expression $\frac{1}{2}\left[\left(q_{11}+q_{12}\right)^{2}+\left(q_{21}+q_{22}\right)^{2}\right]$. Substituting for the equilibrium quantities, we obtain

$$
C(g(12,12))=\frac{4(a-c)^{2}}{9(1+d)^{2}(2-d)^{2}} .
$$

For any distribution network $g$, social or aggregate welfare is defined as the sum of consumer surplus and total equilibrium profits. Let $W(g(12,12))$ denote aggregate welfare in network $g(12,12)$. Then,

$$
W(g(12,12))=\frac{4\left(5+d-3 d^{2}\right)(a-c)^{2}}{9(1+d)^{2}(2-d)^{2}}-8 k
$$

In the appendix we give the equilibrium profits, the sum of the individual equilibrium profits, the consumer surplus and the social welfare for each possible distribution network among the two manufacturers and the two retailers. The other relevant equilibrium variables $q$ 's and $w$ 's are available from the authors upon request.

\section{Stable distribution networks}

A simple way to analyze the networks that one might expect to emerge in the long run is to examine a sort of equilibrium requirement that agents not benefit from altering the structure of the network. A weak version of such condition is the pairwise stability notion defined by Jackson and Wolinsky (1996). A network is pairwise stable if no agent benefits from severing one of their links and no other two agents benefit from adding a link between them, with one benefiting strictly and the other at least weakly.

Definition 1 A network $g$ is pairwise stable if

(i) for all $(i, j) \in g, \Pi_{i}(g) \geq \Pi_{i}(g-(i, j))$ and $\Pi_{j}(g) \geq \Pi_{j}(g-(i, j))$, and 
(ii) for all $(i, j) \notin g$, if $\Pi_{i}(g)<\Pi_{i}(g+(i, j))$ then $\Pi_{j}(g)>\Pi_{j}(g+(i, j))$.

Let us say that $g^{\prime}$ is adjacent to $g$ if $g^{\prime}=g+(i, j)$ or $g^{\prime}=g-(i, j)$ for some $(i, j)$. A network $g^{\prime}$ defeats $g$ if either $g^{\prime}=g-(i, j)$ and $\Pi_{i}\left(g^{\prime}\right) \geq \Pi_{i}(g)$, or if $g^{\prime}=g+(i, j)$ with $\Pi_{i}\left(g^{\prime}\right) \geq \Pi_{i}(g)$ and $\Pi_{j}\left(g^{\prime}\right) \geq \Pi_{j}(g)$ with at least one inequality holding strictly. Pairwise stability is equivalent to saying that a network is pairwise stable if it is not defeated by another (necessarily adjacent) network.

While pairwise stability is natural and quite easy to work with, it is a concept with some limitations. First, it is a weak notion in that it only considers deviations on a single link at a time. For instance, it could be that an agent would not benefit from severing any single link but would benefit from severing several links simultaneously, and yet the network would still be pairwise stable. Second, pairwise stability considers only deviations by at most a pair of agents at a time. It might be that some group of agents could all be made better off by some complicated reorganization of their links, which is not accounted for under pairwise stability. The definition of strong stable networks is in that spirit, and is due to Jackson and van den Nouweland (2005). A strongly stable network is a network which is stable against changes in links by any coalition of agents.

A network $g^{\prime} \in G$ is obtainable from $g \in G$ via deviations by $S \subset N$ if

(i) $i j \in g^{\prime}$ and $i j \notin g$ implies $i j \subset S$, and

(ii) $i j \in g$ and $i j \notin g^{\prime}$ implies $i j \cap S \neq \varnothing$.

The above definition identifies changes in a network that can be made by a coalition $S$, without the need of consent of any agents outside of $S$. Part (i) requires that any new links that are added can only be between agents in $S$. This reflects the fact that consent of both agents is needed to add a link. Part (ii) requires that at least one agent of any deleted link be in $S$. This reflects the fact that either agent in a link can unilaterally sever the relationship.

Definition 2 A network $g$ is strongly stable if for any $S \subset N, g^{\prime}$ that is obtainable from $g$ via deviations by $S$, and $i \in S$ such that $\Pi_{i}\left(g^{\prime}\right)>\Pi_{i}(g)$, there exists $j \in S$ such that $\Pi_{j}\left(g^{\prime}\right)<\Pi_{j}(g)$.

Strong stability provides a powerful refinement of pairwise stability. The concept of strong stability mainly makes sense in smaller network situations where agents have substantial information about the overall structure and potential payoffs and can coordinate 
their actions. ${ }^{15}$ That is, it makes sense to study the stability of distribution networks between manufacturers and retailers in a successive duopoly.

In order to characterize the strongly stable distribution networks we first derive the pairwise stable networks since a strongly stable network is pairwise stable while the reverse is not true. To make meaningful comparisons we impose an upper bound on the link cost $k$ so that for each possible distribution network any equilibrium output and payoffs are positive. The upper bound on $k$ is given in the next lemma and displayed in Figure $2 .{ }^{16}$

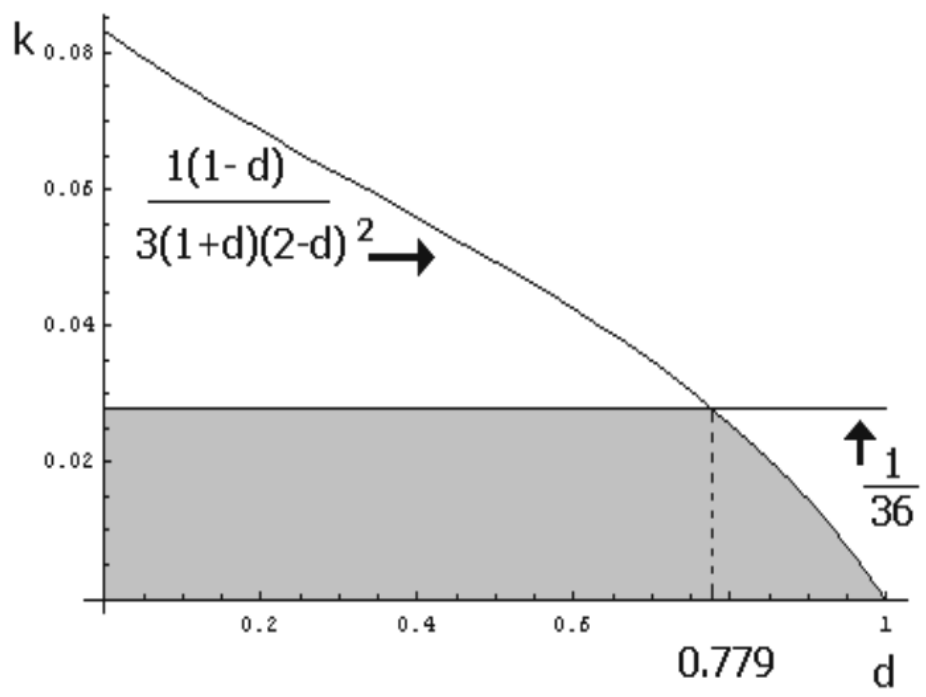

Figure 2: Bounds on the link cost $k$.

Lemma 1 All agents' equilibrium payoffs are positive in each possible distribution network if the link cost $k$ is bounded above as follows, $k \leq \frac{(a-c)^{2}}{36}$ if $0<d \leq 0.779$ and

\footnotetext{
${ }^{15}$ Coordination may be facilitated through industry associations which regroup firms having some common interest within an industry. For instance, the agri-food industry in Canada has 31 industry associations. One of them is the Brewers Association of Canada whose role is to foster and improve business relations and cooperation generally between brewers in Canada and between them and the public in the furtherance and protection of their respective interests and welfare. The assocation provides services to industry including statistics on beer consumption, monitoring government and policy issues, and the promotion of responsible consumption. Another association is the Food and Consumer Products Manufacturers of Canada whose aim is to enhance growth and competitiveness of the food and consumer products manufacturing industry. There is also the Canadian Association of Independent Grocers which is a non-profit trade association founded in 1962 with the purpose of furthering the unique interests of Canada's independently owned and franchised supermarkets. More information can be found at http://www.agr.gc.ca/

${ }^{16}$ In all figures that appear in the paper we have considered the case where $(a-c)=1$.
} 
$k \leq \frac{(1-d)(a-c)^{2}}{3(1+d)(2-d)^{2}}$ if $0.779<d<1$

All proofs can be found in the appendix. Denote the upper bound on $k$ as $\bar{k}$, where

$$
\bar{k} \equiv \min \left\{\frac{(a-c)^{2}}{36}, \frac{(1-d)(a-c)^{2}}{3(1+d)(2-d)^{2}}\right\} .
$$

The first term corresponds to the constraint on $k$ that implies that $\Pi_{R_{1}}(g(1,1))$ is positive while the second is the one that ensures $\Pi_{M_{1}}(g(12,12))>0$. The following remarks are useful in understanding Figure 3 which displays the pairwise stable distribution networks.

a) The distribution network $g(12,12)$ is pairwise stable if and only if $k<\min \left\{k_{(12,12)}^{M}\right.$, $\left.k_{(12,12)}^{R}\right\}$ since no agent wants to sever a link.

b) The distribution network $g(1,2)$ (and $g(2,1)$ ) is pairwise stable if and only if $k>$ $\min \left\{k_{(1,2)}^{M}, k_{(1,2)}^{R}\right\}$ since no pair manufacturer-retailer wants to create a link and no agent wants to destroy a link.

c) The distribution network $g(12,1)$ (and $g(12,2), g(1,12)$ and $g(2,12)$ ) is pairwise stable if and only if $k>\min \left\{k_{(12,12)}^{M}, k_{(12,12)}^{R}\right\}$ and $k<\min \left\{k_{(1,2)}^{M}, k_{(1,2)}^{R}\right\}$ since no agent wants to sever a link and the pair manufacturer-retailer with only one link does not want to create another link.

Therefore, in the area $\mathrm{C}$ the only pairwise stable distribution network is $g(1,2)$; in the area B both $g(1,2)$ and $g(12,12)$ are pairwise stable; in the area A only $g(12,12)$ is pairwise stable; and in the area D $g(12,1)$ is the only pairwise stable distribution network. The following proposition summarizes pairwise stability among distribution networks.

Proposition 1 Only three distribution networks can be pairwise stable: $g(12,12), g(12,1)$ and $g(1,2)$. The parameter space $(k, d)$ is partitioned into four regions. In the first region, for low values of $d, g(12,12)$ is the only pairwise stable network. In the second, for low values of $d$ and intermediate values of $k, g(12,1)$ is the only pairwise stable network. In the third region, for high and low values of $d$ and values of $k$ close to the upper bound, $g(1,2)$ is the only pairwise stable network. In the fourth region, for intermediate values of $d$, there are two pairwise stable networks $g(12,12)$ and $g(1,2)$.

It is interesting to note that when $k=0$, only two distribution networks are pairwise stable: ${ }^{17}$ the non-exclusive distribution \& non-exclusive dealing system $g(12,12)$ and

\footnotetext{
${ }^{17}$ For $k=0$ (assumption made in Mycielski, Riyanto and Wuyts (2000) and Moner-Colonques, SempereMonerris and Urbano (2004)), the pairwise stable distribution networks coincide exactly with the subgame perfect Nash equilibrium distribution systems of Moner-Colonques, Sempere-Monerris and Urbano (2004) when brands are symmetric:
} 


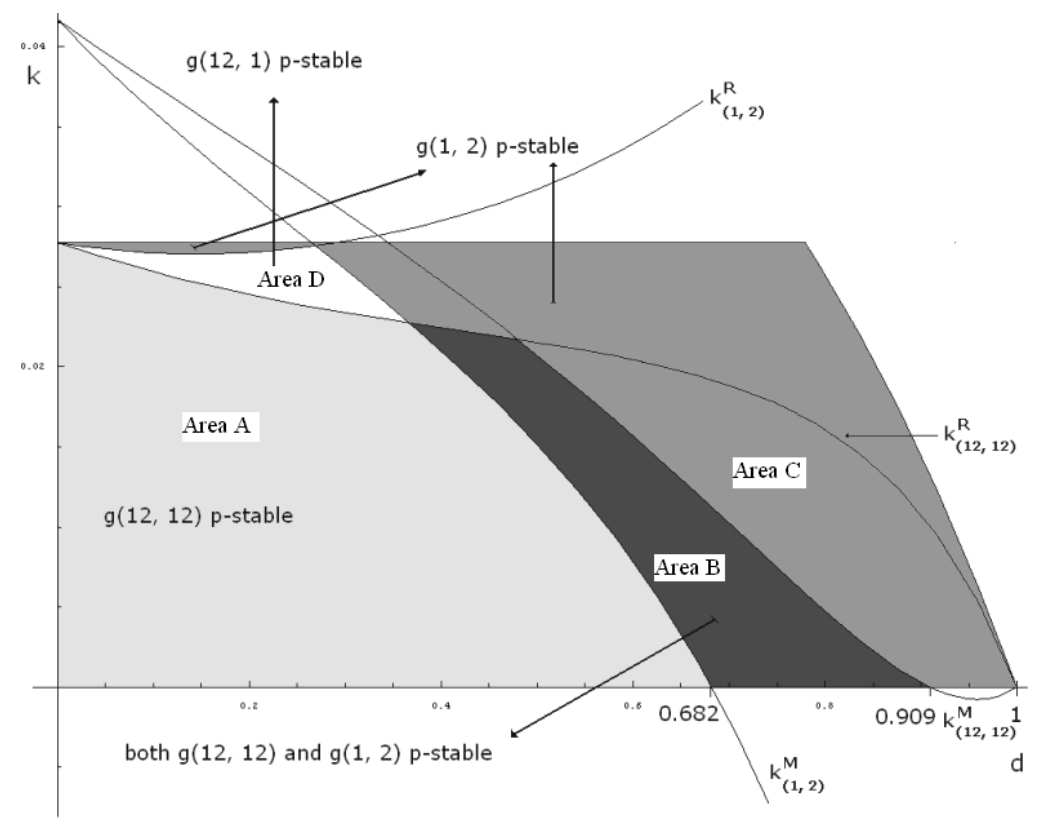

Figure 3: Pairwise stability of distribution networks.

the exclusive distribution \& exclusive dealing system $g(1,2) ; g(12,12)$ is stable when the products are sufficiently differentiated $d \in(0,0.682)$, both $g(12,12)$ and $g(1,2)$ are stable for intermediate values $d \in(0.682,0.909)$, and finally, $g(1,2)$ is stable when products are close substitutes $d \in(0.909,1) .{ }^{18}$ In the absence of link costs, the pairwise stability of a network depends on the strategic incentives of manufacturers and retailers to introduce intrabrand competition once there is interbrand competition. Take the distribution network $g(1,2)$. A manufacturer and a retailer would like to form a link between them only if the degree of interbrand rivalry is not too high; that is, if and only if $d \in(0,0.682)$. Thus, for $d \in(0,0.682)$, the distribution network $g(1,2)$ is defeated by the network $g(12,2)$. Take now the distribution network $g(12,2)$. In order for $g(12,2)$ to be stable, the pair formed by $M_{1}$ and $R_{2}$ should have no interest in adding a link between them. But, given the low degree of interbrand rivalry, $d \in(0,0.682)$, they also prefer to establish a link between them. Thus, for $d \in(0,0.682)$, the distribution network $g(1,2)$ is defeated by the network $g(12,2)$ and $g(12,2)$ is defeated by $g(12,12)$. Observe that $g(12,2)$ is defeated by $g(12,12)$ not only for these values of interbrand rivalry but also for $d \in(0.682,0.909)$.

\footnotetext{
${ }^{18}$ When joint consent is needed to establish and/or maintain a link between a manufacturer and a retailer, the asymmetric distribution network $g(12,2)$ is no more stable. In Moner-Colonques, SempereMonerris and Urbano (2004) this network appears at equilibrium for sufficiently large brand asymmetry. In Mycielski, Riyanto and Wuyts (2000), $g(12,2)$ is an equilibrium outcome when goods are strong substitutes but not perfect ones. Here only for positive link costs, one can sustain $g(12,2)$ as pairwise stable.
} 
Once the formation of links is costly, the pairwise stability of a given distribution network also depends on the size of link costs. In such case, the incentives to add a link between a manufacturer and a retailer when the degree of interbrand rivalry is low can be offset by the negative effect of the costly link on profits. When it happens, the distribution networks $g(12,2)$ and $g(1,2)$ can be pairwise stable for low values of interbrand rivalry; see Figure 3.

Now, we turn to the characterization of strongly stable distribution networks. We already know that the only pairwise stable distribution networks are $g(1,2), g(12,1)$ and $g(12,12)$. To check for strong stability we have to examine the incentives that a coalition of agents have to move from the pairwise stable networks to other networks. Specifically,

a) In considering the strong stability of $g(1,2)$, we have to check for the incentives to move from $g(1,2)$ to $g(12,12)$, next to $g(12,0)$, and then to $g(1,1)$.

b) In considering the strong stability of $g(12,12)$, we have to check for the incentives to move from $g(12,12)$ to $g(1,2)$, next to $g(12,0)$, and then to $g(1,1)$.

c) In considering the strong stability of $g(12,1)$, we have to check for the incentives to move from $g(12,1)$ to $g(1,0)$.

Proposition 2 The distribution network $g(1,2)$ is always strongly stable when it is pairwise stable. However, the distribution networks $g(12,1)$ and $g(12,12)$ are not necessarily strongly stable when they are pairwise stable.

Figure 4 displays the areas in the space $(k, d)$ where the three distribution networks are strongly stable or pairwise stable. It is interesting to note that for $k=0$, the non-exclusive distribution \& non-exclusive dealing system $g(12,12)$ is strongly stable when the products are rather differentiated $d \in(0.202,0.510)$, and the exclusive distribution \& exclusive dealing system $g(1,2)$ is strongly stable for $d \in(0.682,1)$. Contrary to the case of pairwise stability, there are some values of product differentiation for which no network is strongly stable. This happens because $g(12,12)$ is defeated by $g(1,2)$ either when the products are very differentiated (for $d \in(0,0.202)$ ) or for intermediate levels of product differentiation (for $d \in(0.510,0.682))$. In fact, when products are very differentiated, $d \in(0,0.202)$, the coalition of two retailers prefers to delete two links, one with each manufacturer, in order to form a differentiated duopoly without intrabrand rivalry (a situation close to two successive monopolies). For intermediate levels of $d \in(0.510,0.682)$ the coalition of two manufacturers prefers to delete two links, one with each retailer, in order to form a differentiated duopoly in the distribution market and avoiding introducing intrabrand rivalry. Thus, when the degree of product differentiation is high enough or intermediate, 


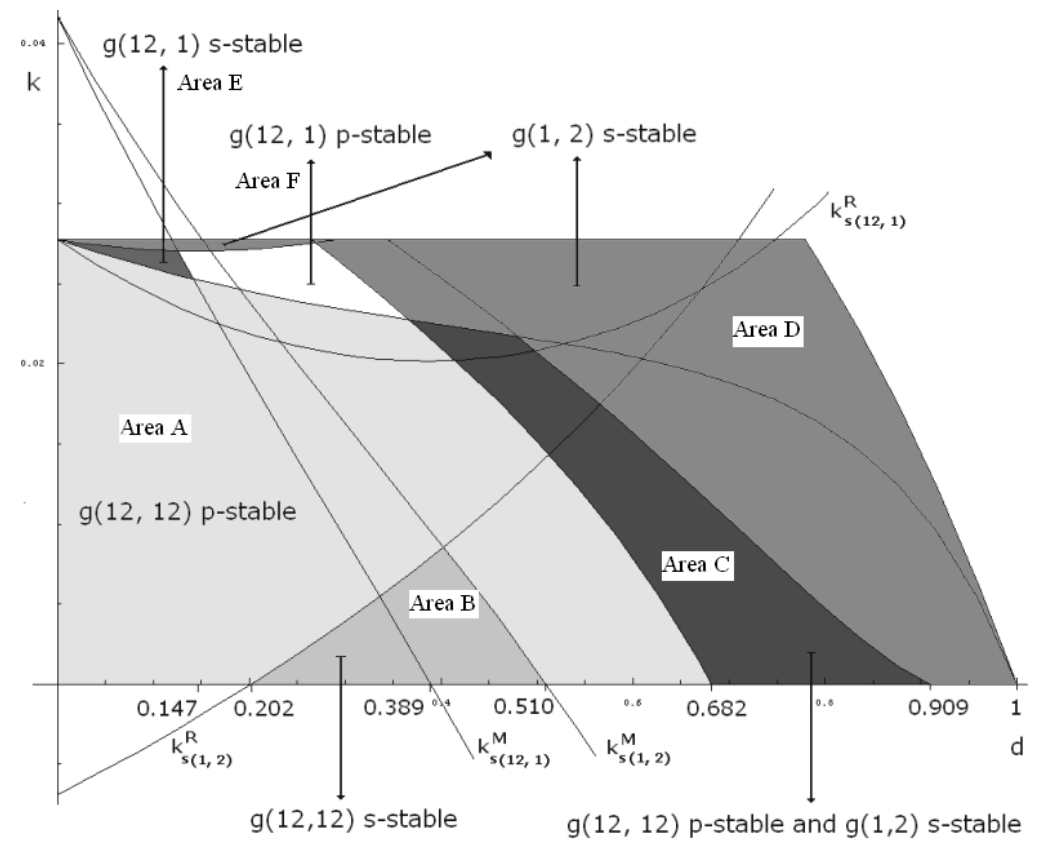

Figure 4: Strongly stable and pairwise stable distribution networks.

the non-exclusive distribution \& non-exclusive dealing system will not emerge in the "longrun" while Mycielski, Riyanto and Wuyts (2000) and Moner-Colonques, Sempere-Monerris and Urbano (2004) have shown that it is a "short-run" equilibrium.

Once the formation of links is costly, the stability of a given distribution network also depends on the size of link costs. In such a case, the incentives to delete links by the coalition of two retailers when the degree of interbrand rivalry is very low, or by the coalition of the two manufacturers when the degree of interbrand rivalry is intermediate can be reinforced by the negative effect of the costly links on profits. There is a size of link costs from which the distribution network $g(12,12)$ is no longer strongly stable. Higher size of link costs and low degree of interbrand rivalry make the distribution networks $g(12,1)$ and $g(1,2)$ strongly stable. But contrary to the case of pairwise stability, the possibility that $M_{1}$ and $R_{1}$ delete respectively their links with $R_{2}$ and $M_{2}$ moving to $g(1,0)$, makes $g(12,1)$ no longer strongly stable for some values of $d$ and $k$ for which it was pairwise stable. For high degree of interbrand rivalry, the fact of costly links does not prevent the strong stability of $g(1,2)$. In Figure 4, one can observe that while there are always at least a pairwise stable network, for some values of the degree of product differentiation and link costs there is no strongly stable network.

When no network is strongly stable we will observe a sequence of distribution networks due to continuously profitable deviations. In terms of competition policy, it would be 
interesting to know which networks are likely to be visited by such sequence of profitable deviations. In fact we will show that some distribution networks will be visited at most once, while others will belong to a closed cycle and will be visited regularly. We now define what is meant by a closed cycle. A network $g^{\prime}$ strongly defeats $g$ if (i) $g^{\prime}$ is obtainable from $g$ via deviations by $S \subset N$ and (ii) $\Pi_{i}\left(g^{\prime}\right) \geq \Pi_{i}(g)$ for all $i \in S$ and $\Pi_{j}\left(g^{\prime}\right)>\Pi_{j}(g)$ for some $j \in S$. An improving path from a network $g$ to a network $g^{\prime}$ is a finite sequence of graphs $g_{1}, g_{2}, \ldots, g_{K}$ with $g_{1}=g$ and $g_{K}=g^{\prime}$ such that for any $k \in\{1, \ldots, K-1\}$ we have $g_{k+1}$ strongly defeats $g_{k}$. A set of networks $\bar{G}$ form a cycle if for any $g \in \bar{G}$ and $g^{\prime} \in \bar{G}$ there exists an improving path connecting $g$ to $g^{\prime}$. A cycle $\bar{G}$ is a closed cycle if no network in $\bar{G}$ lies on an improving path leading to a network that is not in $\bar{G}$. In characterizing the closed cycles (whose proof is given in the appendix) we distinguish two cases:

a) If $\min \left\{\min \left\{k_{(12,12)}^{R}, k_{(1,2)}^{M}\right\}, \max \left\{k_{s(12,1)}^{M}, k_{s(12,1)}^{R}\right\}\right\}>k>\min \left\{k_{s(1,2)}^{M}, k_{s(1,2)}^{R}\right\}$, then there is a unique closed cycle which consists of networks $g(12,12), g(1,2), g(2,1)$, $g(12,1), g(12,2), g(1,12)$ and $g(2,12)$.

b) If $\min \left\{k_{(1,2)}^{R}, k_{(1,2)}^{M}\right\}>k>\max \left\{k_{s(12,1)}^{M}, k_{s(12,1)}^{R}\right\}$, then there is a unique closed cycle which consists of all possible distribution networks.

Figure 5 displays the areas in the space $(k, d)$ where there is no strongly stable network (areas $\mathrm{A}$ and $\mathrm{E}$ ) and where some of the three distribution networks are strongly stable. In area A no strongly stable network exists and there is a unique closed cycle which consists of networks $g(12,12), g(1,2), g(2,1), g(12,1), g(12,2), g(1,12)$ and $g(2,12)$. In area $\mathrm{E}$ no strongly stable network exists and there is a unique closed cycle which consists of all possible networks. In areas $\mathrm{B}, \mathrm{C}$ and $\mathrm{D}$ the distributions networks $g(12,12), g(1,2)$ and $g(12,1)$ are, respectively, the unique strongly stable distribution network. Thus, when the degree of differentiation is high and link costs are not too high or when the degree of differentiation is intermediate and link costs are small (area A in Figure 5) we will observe a cycle of distribution networks where non-exclusive distribution \& non-exclusive dealing will succeed to mixed distribution system, exclusive distribution \& exclusive dealing will succeed to non-exclusive distribution \& non-exclusive dealing, and mixed distribution system will succeed to exclusive distribution \& exclusive dealing. Moreover, there is no cycle beside the closed one and networks outside the closed cycle will be visited at most once. So, from any other distribution networks all sequences of profitable deviations long enough go to the closed cycle. 


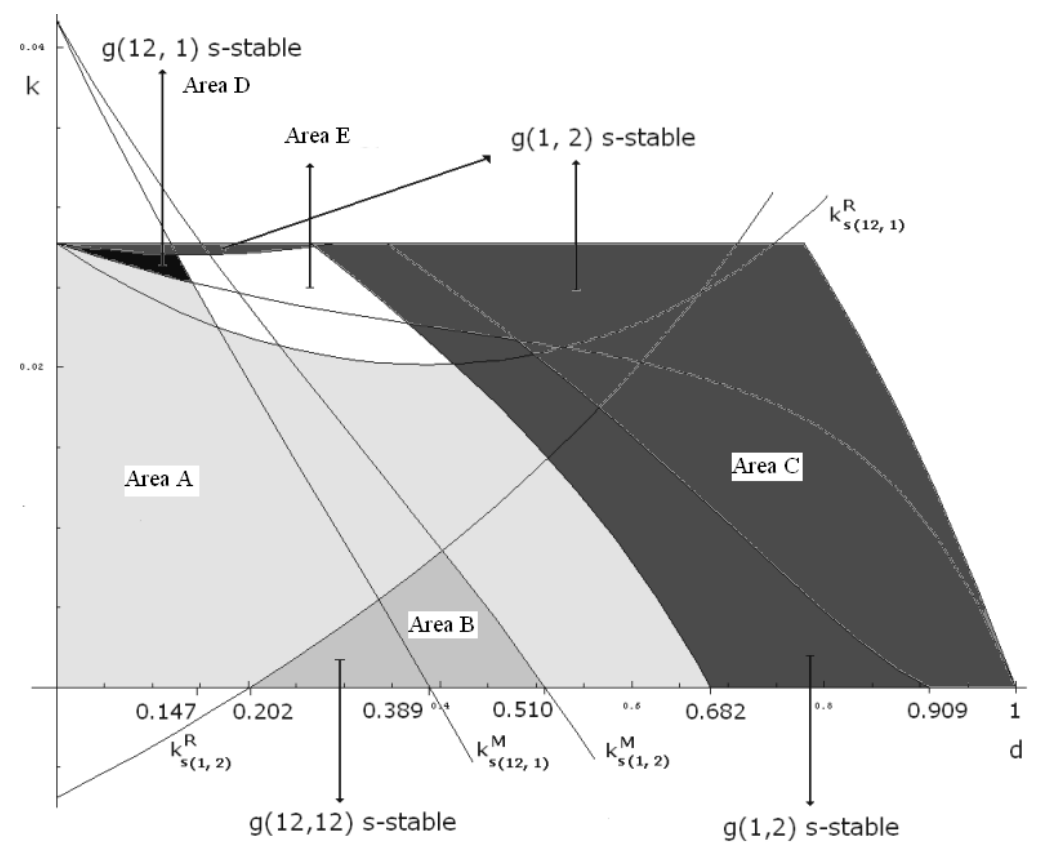

Figure 5: Cycles and strongly stable networks.

\section{Efficiency, consumer surplus and social welfare}

Some of the very central questions about network formation concern the conditions under which the networks which are formed by the players turn out to be efficient from an overall societal perspective. In order to discuss these issues we need to define what is meant by efficiency. The network structure is the key determinant of the level of productivity or utility to the society of players involved. In our case, a manufacturer's expected profit and a retailer's expected profit from establishing a link among them in order to sell the manufacturer's brand to consumers depend on how many links each of them has formed and on how many links the other manufacturer and retailer have established. Remember that $\Phi$ is a function that assigns to each network $g$ a value $\Phi(g)$ that represents the overall total value of network $g$ which is the sum of the equilibrium profits of the four agents.

An obvious notion of efficiency is simply maximizing the overall total value among all possible networks. This notion was referred to as strong efficiency by Jackson and Wolinsky (1996), but we will simply refer to it as efficiency.

Definition 3 A network $g$ is efficient relative to $\Phi$ if $\Phi(g) \geq \Phi\left(g^{\prime}\right)$ for all $g^{\prime} \in G$.

It is clear that there will always exist at least one efficient network, given that there is only a finite set of networks. A starting point is to examine efficiency when the cost of the links is negligible. 
Proposition 3 Suppose that links are costless, $k=0$. Then, the efficient distribution network is $g(12,12)$ for $0<d \leq 0.735, g(12,1)$ for $0.735<d \leq 0.863$, and $g(12,0)$ for $0.863<d<1$.

In the absence of costly links, the degree of product differentiation determines the stable networks but also the more profitable network from the manufacturers and retailers point of view. A low degree of product differentiation implies a more competitive environment and thus manufacturers (and retailers) prefer a distribution network without intrabrand competition. Since the transfer price under $g(12,0)$ is smaller than the transfer price under $g(1,2)$, the distribution network with a unique multiproduct retailer $g(12,0)$ is more efficient than the distribution network with two differentiated retailers $g(1,2)$. Higher degrees of product differentiation will give incentives to one manufacturer to use two retailers making $g(12,1)$ the new efficient network. Further increases in the degree of product differentiation will now give incentives to the second manufacturer to use two retailers too. Since the output expansion effect dominates the competition effect, $g(12,12)$ becomes the new efficient distribution network.

We now analyze efficiency for positive link costs. Positive link costs adds a second negative effect to the formation of a new link. For that reason, the distribution networks $g(12,12)$ and $g(12,1)$ are no more efficient for high enough link costs. Only $g(1,2)$ and $g(12,0)$ are efficient for high sizes of link costs. As the degree of product differentiation increases, the exclusive distribution \& exclusive dealing network $g(1,2)$ will lead to higher aggregate profits than the exclusive distribution \& non-exclusive dealing network $g(12,0)$ with in-store interbrand competition. Indeed, the difference in transfer prices is compensated by the increased degree of product differentiation. Figure 6 displays the efficient distribution networks. The network $g(12,12)$ is the efficient one in the area $\mathrm{A} ; g(1,2)$ is the efficient network in the area $\mathrm{C} ; g(12,1)$ is the efficient network in the area $\mathrm{B}$; and $g(12,0)$ is the efficient network in the area D. The next proposition summarizes the main interesting features about efficient distribution networks when links are costly. The complete characterization of the efficient distribution networks is given in Proposition 7 of the appendix.

Proposition 4 Suppose that links are costly, $k>0$. Any distribution network marketing two products is efficient under particular conditions on the degree of product differentiation and the size of link costs.

The distribution network $g(12,12)$ is efficient for low enough link cost and enough degree of product differentiation. As link costs increase the efficient distribution network is either $g(1,2)$, for high and intermediate degrees of product differentiation, or $g(12,1)$ for lower 
degrees of product differentiation. Further increases in link costs and intermediate degrees of product differentiation imply that the efficient distribution network is either $g(1,2)$ or $g(12,0)$. Finally, for small enough degrees of product differentiation the efficient network is $g(12,0)$.

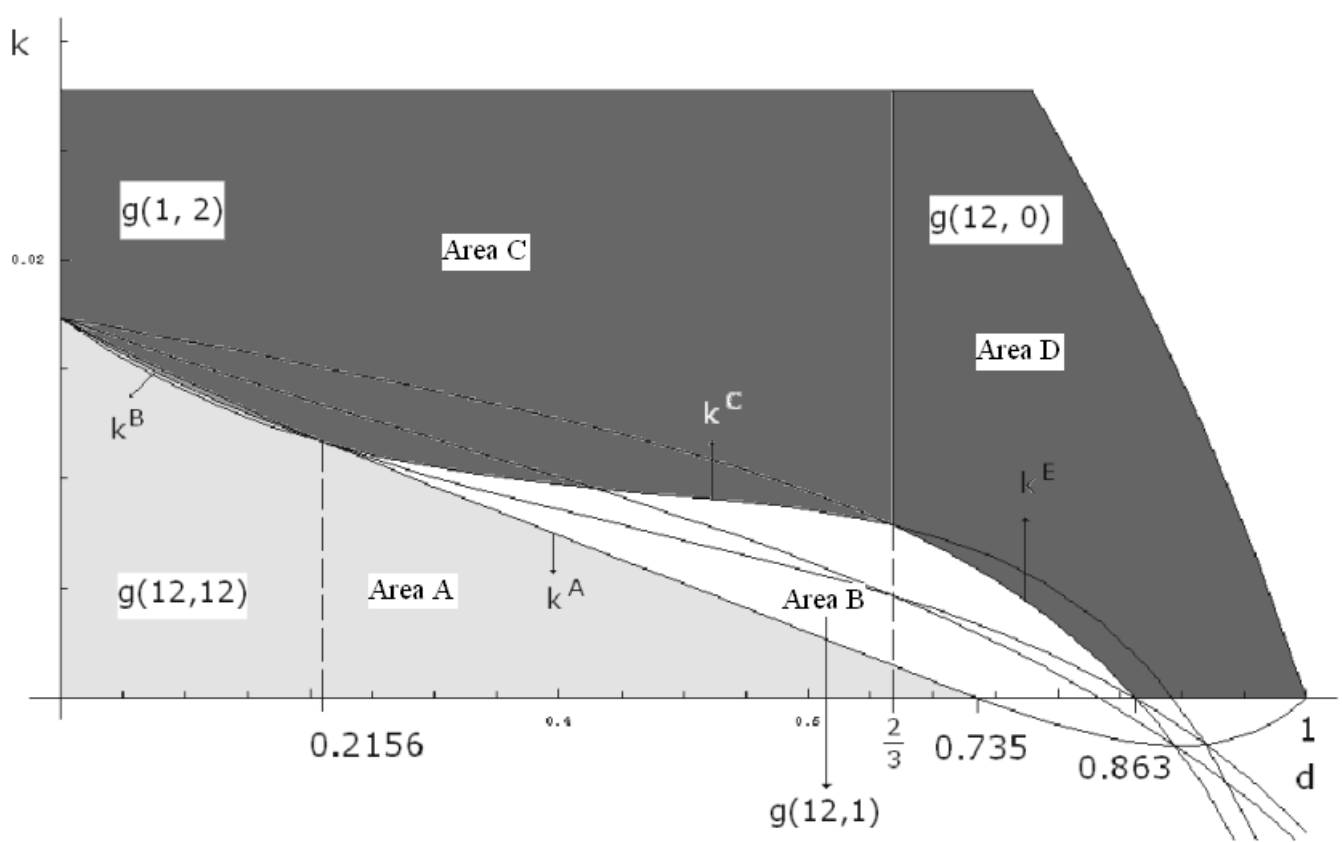

Figure 6: Efficient distribution networks.

There is no coincidence between the set of efficient networks and that of pairwise or strongly stable distribution networks. We find that the three stable networks can be efficient for particular values of the degree of product differentiation and link costs, but not necessarily for the values under which they are stable. Moreover, the network $g(12,0)$ is efficient for large enough link costs and low enough product differentiation but it is never stable.

Before analyzing the social welfare implications of the different distribution networks, it is worthy to study how consumer surplus is affected. Depending on the distribution network, one or two products are present in the market. Different combinations of intrabrand, interbrand and in-store competition can be present. Which distribution network will give the highest consumer surplus?

Proposition 5 The highest level of consumer surplus is achieved when the distribution network $g(12,12)$ is formed. Moreover, consumer surplus is increasing with the introduction of product items in the outlets. 
Consumers prefer that the three types of competition are present in both outlets, that is two product items in the two outlets. If this is not possible, they prefer one retailer with in-store competition and the other not, but with the presence of both inter and intrabrand competition, that is one outlet with one product item and the other with two products. If the latter two options are not possible, consumers prefer at least two product items, either two different products concentrated in one outlet for low enough product differentiation, for $0.8597<d<1$, or the same product item in each of the outlets for $0.2826<d<0.8597$, or finally, two different product items each one in a different outlet, for $0<d<0.2826$. The consumer surplus depends on both the number of product items and its distribution among outlets. When the number of product items is the same, then its distribution also affects the degree of competition among manufacturers and among retailers. For example, in the distribution network $g(12,0)$ although there is only one retailer the rivalry among manufacturers implies that the transfer price is lower than the transfer price in a network where only one product is present in the market, as for example $g(1,1)$. In contrast, in the latter distribution network the rivalry among retailers is higher than in the former. The combination of both effects explains why $g(12,0)$ generates a higher consumer surplus than $g(1,1)$ when $d$ is large enough. ${ }^{19}$ Finally, the worst distribution network for consumers is the one with only one product item in a unique outlet. The complete characterization of consumers preferences over distribution networks at equilibrium can be found in the appendix.

Finally, we analyze the effects of the different distribution networks on social welfare. We give in the text the main features while the complete characterization is relegated to the appendix. The distribution networks that attain the maximum social welfare are $g(1,2)$ or $g(12,0)$ or $g(12,1)$ or $g(12,12)$, depending on both the degree of product differentiation and the size of the link costs. Thus, only distribution networks that market both products can achieve the highest social welfare. That is, interbrand competition is always socially desirable in the market. However, intrabrand competition and in-store competition are not necessarily socially desirable.

Proposition 6 The distribution network that attains the highest level of social welfare is always one that markets both products. In particular the highest social welfare is obtained with

a) $g(12,12)$ for high enough degrees of product differentiation and low enough link costs. Regardless of the size of $k, g(12,12)$ maximizes social welfare for $0<d \leq 0.0326$.

\footnotetext{
${ }^{19}$ The equilibrum transfer price ranking is: $w(g(12,12))=w(g(12,0))<w_{2}(g(12,1))<w_{1}(g(12,1))<$ $w(g(1,2))<w(g(1,1))=w(g(1,0))$.
} 
b) $g(12,1)$ for low enough degrees of product differentiation and intermediate sizes of link costs. Regardless of the size of $k, g(12,1)$ maximizes social welfare for $d \geq 0.954$.

c) $g(1,2)$ for intermediate degrees of product differentiation, $0.0326<d<\frac{2}{3}$, and high sizes of link costs.

d) $g(12,0)$ for intermediate degrees of product differentiation, $\frac{2}{3}<d<0.880$ and high sizes of link costs.

Figure 7 displays the distribution networks that maximize social welfare. The area $\mathrm{A}$ corresponds to the range of parameters where $g(12,12)$ maximizes social welfare; in the area $\mathrm{C} g(1,2)$ maximizes social welfare; in the area $\mathrm{D} g(12,0)$ maximizes social welfare, and in the area B $g(12,1)$ maximizes social welfare. Comparing Figure 5 with Figure 7 we observe that the distribution networks that firms will endogenously form following their own interest enter, in general, in contradiction with those that maximize welfare.

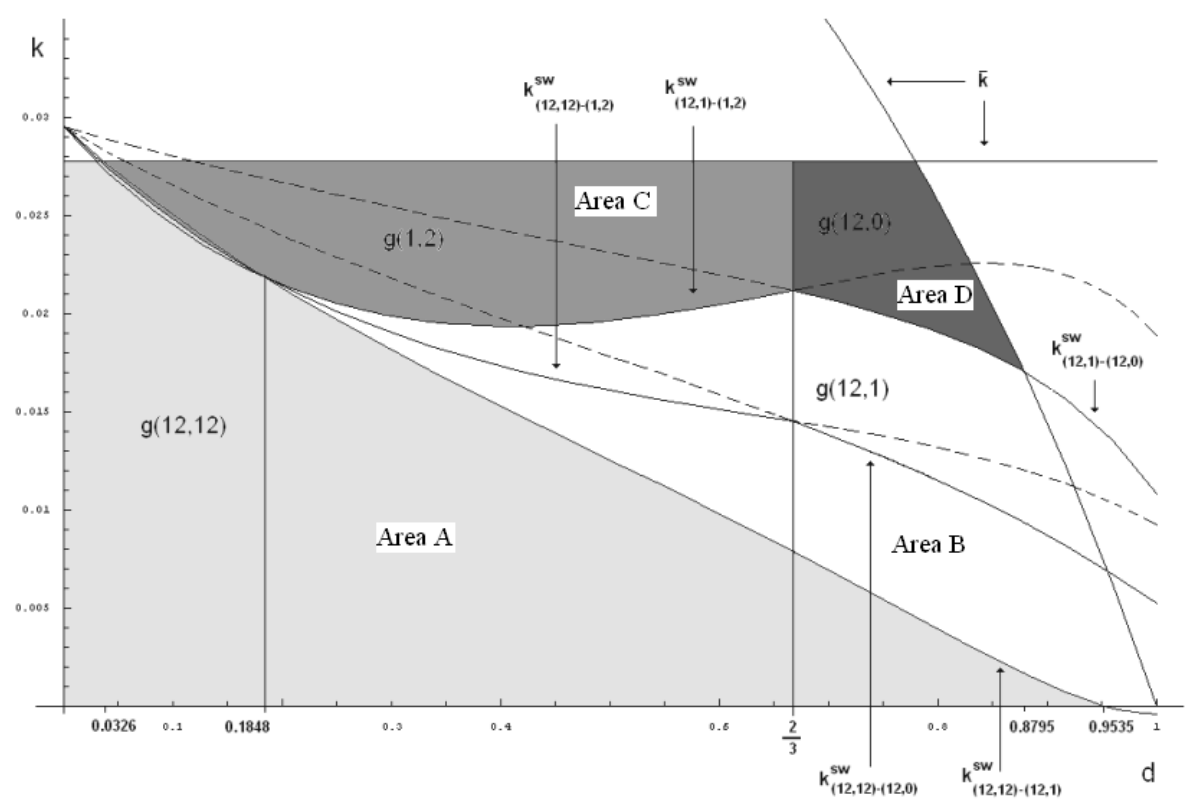

Figure 7: Social welfare maximizing distribution networks.

For the particular case $k=0$, we find that: (i) when $g(12,12)$ is strongly stable it is the one that maximizes social welfare, but the reverse is not true; (ii) $g(12,1)$ maximizes social welfare when $d \in(0.954,1)$ but it is not strongly stable. Once the formation of links is costly, $g(1,2)$ and $g(12,0)$ also maximize social welfare for some values of the degree of product differentiation and of the link costs. While $g(1,2)$ could be strongly stable when it maximizes social welfare, the networks $g(12,0)$ and $g(12,1)$ are never stable when they 
reach the maximum welfare. Moreover, costly links increase the conflict between social welfare and strong stability with respect to the network $g(12,12)$.

In general, there are distribution networks like $g(12,12), g(12,0)$ and $g(12,1)$ that are less likely to arise when leaving the market forces on their own, as compared with the socially desirable outcome. By contrast, the distribution network $g(1,2)$ appears to be stable under more situations than what would be socially desirable. Mycielski, Riyanto and Wuyts (2000) have studied the welfare implications of manufacturers' choices of vertical arrangements and its policy implications in a setting where retailers compete à la Bertrand. They have shown that, for a low degree of interbrand rivalry, any policy measure to restrict vertical restraints is unnecessary. Such policies become necessary once there is a high degree of interbrand rivalry. Their results contrast with the ones we obtain in a setting where retailers compete à la Cournot. Even for costless links, we get that such policies are also necessary for high degrees of product differentiation. Then, restricting exclusive distribution and exclusive dealing arrangements might have a positive impact on social welfare. Impeding the profitable deviation of both retailers from $g(12,12)$ to $g(1,2)$ would make strongly stable the distribution network $g(12,12)$, which is the one that maximizes social welfare.

\section{Conclusion}

We have analyzed the networks between two manufacturers of differentiated goods and two multi-product retailers that one might expect to emerge in the long run. We have found that only three distribution networks are strongly stable for particular values of the degree of product differentiation and link costs. A first distribution network, the nonexclusive distribution \& non-exclusive dealing system, in which both retailers distribute both products is strongly stable for intermediate degrees of product differentiation and small link costs. In this distribution network, both interbrand and intrabrand competition are present in the market. A second distribution network, the exclusive distribution \& exclusive dealing system, in which each retailer distributes a different product is strongly stable for low degrees of product differentiation. In this distribution network, no intrabrand competition appears in the market. A third distribution, the mixed distribution system, in which one retailer distributes both products while the other retailer sells only one is strongly stable for high degrees of product differentiation and large link costs. ${ }^{20}$

\footnotetext{
${ }^{20}$ The mixed distribution system seems quite common in the beer industry. The analysis made by Slade (1998) for the U.K. beer industry reveals that one of the effects of the U.K. Monopolies and Mergers Commission report and the Beer Orders passed after 1989 was the formation of public-house chains which most often operate under exclusive purchasing contracts with major brewers. In $1994,54 \%$ of the public
} 
Finally, for some values of the degree of product differentiation and link costs, no distribution network is strongly stable. In particular, when the degree of product differentiation is high enough or intermediate and link costs are moderate, the non-exclusive distribution \& non-exclusive dealing system will not emerge in the "long-run" in contrast with Mycielski, Riyanto and Wuyts (2000) and Moner-Colonques, Sempere-Monerris and Urbano (2004). However, we will observe a cycle among the above three distribution networks. This is consistent with the observation that the distribution chains organization differs across markets and industries over time. ${ }^{21}$

Consumers are better off in a market with interbrand and intrabrand competition in both products. Thus, they prefer the non-exclusive distribution \& non-exclusive dealing system. We have also investigated whether the stable distribution network, when it exists, maximizes social welfare. When link costs are small enough, two distribution networks may maximize social welfare. The non-exclusive distribution \& non-exclusive dealing system maximizes social welfare if the degree of product differentiation is high enough; otherwise, the mixed distribution system maximizes social welfare. When link costs become large, two other distribution networks may maximize welfare. The exclusive distribution \& exclusive dealing system maximizes welfare if the degree of product differentiation is high enough; otherwise, the exclusive distribution \& non-exclusive dealing system maximizes welfare. Thus, a conflict between stability and social welfare is likely to occur, even more if the degree of product differentiation is either low or high. This conflict is crucial from a competition policy perspective and is summarized in Table 1.

\section{Acknowledgments}

We wish to thank Paul Belleflamme, Rafael Moner-Colonques and Xavier Wauthy for helpful comments and discussion. This research started when Ana Mauleon was at LABORES

houses were owned by either national or regional and local brewers, $27 \%$ corresponded to free pubs while the new formed public-chains accounted for the $19 \%$ of 56675 total number of public houses. The free pubs sell the beer of several brewers while the tied pubs are exclusive. Thus, there is evidence that exclusive retailers compete with multi-brand ones in the U.K. beer industry. For the U.S. beer industry, Sass (2005) has found evidence of a mixed pattern of distribution. All major U.S. brewers employ a mix of exclusive and non-exclusive distributors for their products. In particular, 38.7\%. of Anheuser-Busch distributors were exclusive, while $98,3 \%$ of Miller and Coors distributors were non-exclusive. So, U.S. beer industry is another example of coexistence of exclusive retailers with multi-brand ones.

${ }^{21}$ Examples of industries where products are differentiated and are sold by several multi-product retailers are books, TV sets, cola carbonated drinks; where products are differentiated but sold mainly by exclusive retailers are cars; where products are not very differentiated and sold by exclusive retailers are gas; and where products are differentiated and sold by both exclusive and multi-brand retailers are beer, industrial machinery/equipment and electronic and electric equipment. 


\begin{tabular}{|c|c|c|c|c|}
\hline & \multirow{2}{*}{$\begin{array}{l}\text { Link } \\
\text { cost }\end{array}$} & \multicolumn{3}{|c|}{ Product differentiation } \\
\hline & & high & medium & low \\
\hline \multirow{3}{*}{$\begin{array}{l}\text { Strong } \\
\text { stability }\end{array}$} & small & cycle & $\begin{array}{c}\text { Non-excl. distribution } \\
\& \text { non-excl. dealing, } \\
\text { or cycle }\end{array}$ & $\begin{array}{l}\text { Excl. distribution } \\
\text { \& excl. dealing }\end{array}$ \\
\hline & medium & cycle & cycle & $\begin{array}{l}\text { Excl. distribution } \\
\text { \& excl. dealing }\end{array}$ \\
\hline & high & $\begin{array}{l}\text { Excl. distribution } \\
\& \text { excl. dealing, } \\
\text { or cycle, } \\
\text { or mixed system }\end{array}$ & $\begin{array}{l}\text { Excl. distribution } \\
\text { \& excl. dealing, } \\
\text { or cycle }\end{array}$ & $\begin{array}{l}\text { Excl. distribution } \\
\text { \& excl. dealing }\end{array}$ \\
\hline \multirow{3}{*}{$\begin{array}{l}\text { Social } \\
\text { welfare }\end{array}$} & small & $\begin{array}{l}\text { Non-excl. distribution } \\
\text { \& non-excl. dealing }\end{array}$ & $\begin{array}{l}\text { Non-excl. distribution } \\
\text { \& non-excl. dealing }\end{array}$ & $\begin{array}{c}\text { Non-excl. distribution } \\
\& \text { non-excl. dealing, } \\
\text { or mixed system }\end{array}$ \\
\hline & medium & $\begin{array}{l}\text { Non-excl. distribution } \\
\& \text { non-excl. dealing }\end{array}$ & $\begin{array}{l}\text { Non-excl. distribution } \\
\text { \& non-excl. dealing, } \\
\text { or mixed system }\end{array}$ & Mixed system \\
\hline & high & $\begin{array}{l}\text { Non-excl. distribution } \\
\text { \& non-excl. dealing, } \\
\text { or excl. distribution } \\
\quad \& \text { excl. dealing }\end{array}$ & $\begin{array}{l}\text { Excl. distribution } \\
\text { \& excl. dealing }\end{array}$ & $\begin{array}{l}\text { Excl. distribution } \\
\text { \& non-excl. dealing }\end{array}$ \\
\hline
\end{tabular}

Table 1: The conflict between stable distribution networks and social welfare 
(URA 362 CNRS). Financial support from Spanish Ministerio de Ciencia y Tecnología under the projects BEC2000-1429 and BEC 2003-02084, support from Spanish Ministerio de Educación y Ciencia under the project SEJ2004-07554/ECON, support fron the Generalitat Valenciana under the project GRUPOS04/13, support from the Belgian French Community's program Action de Recherches Concertée 03/08-302 (UCL), support of a SSTC grant from the Belgian Federal government under the IAP contract 5/26 (FUSL), and support from the CNRS under the project GW/SCSHS/SH/2003-41 are gratefully acknowledged.

This paper presents research results of the Belgian Program on Interuniversity Poles of Attraction initiated by the Belgian State, Prime Minister's Office, Science Policy Programming.

\section{Appendix}

\section{A Manufacturers and retailers payoffs}

We give the payoffs of the different possible distribution networks between two manufacturers and two retailers. Given the symmetry of the model, only six different distribution networks are at play. Apart from the distribution network $g(12,12)$ already examined in Section 2, the other distribution networks are as follows.

a) The distribution network $g(1,0)$ is symmetric to the distribution networks $g(0,1)$, $g(2,0)$ and $g(0,2)$. There is a successive monopoly in the market: one manufacturer sells its product through a single retailer. Agents' payoffs, its sum, consumer surplus and social welfare are:

$$
\begin{aligned}
\Pi_{M_{1}}(g(1,0)) & =\Pi_{M_{1}}(g(2,0))=\Pi_{M_{2}}(g(0,1))=\Pi_{M_{2}}(g(0,2)) \\
& =\frac{(a-c)^{2}}{8}-k \\
\Pi_{R_{1}}(g(1,0)) & =\Pi_{R_{1}}(g(2,0))=\Pi_{R_{2}}(g(0,1))=\Pi_{R_{2}}(g(0,2)) \\
& =\frac{(a-c)^{2}}{16}-k \\
\Phi(g(1,0)) & =\Phi(g(2,0))=\Phi(g(0,1))=\Phi(g(0,2))=\frac{3(a-c)^{2}}{16}-2 k \\
C(g(1,0) & =C(g(2,0))=C(g(0,1))=C(g(0,2))=\frac{(a-c)^{2}}{32} \\
W(g(1,0) & =W(g(2,0))=W(g(0,1))=W(g(0,2))=\frac{7(a-c)^{2}}{32}-2 k
\end{aligned}
$$


b) The distribution network $g(1,1)$ is symmetric to $g(2,2)$.

$$
\begin{aligned}
\Pi_{M_{1}}(g(1,1)) & =\Pi_{M_{2}}(g(2,2))=\frac{(a-c)^{2}}{6}-2 k \\
\Pi_{R_{1}}(g(1,1)) & =\Pi_{R_{2}}(g(1,1))=\Pi_{R_{1}}(g(2,2))=\Pi_{R_{2}}(g(2,2)) \\
& =\frac{(a-c)^{2}}{36}-k \\
\Phi(g(1,1)) & =\Phi(g(2,2))=\frac{2(a-c)^{2}}{9}-4 k \\
C(g(1,1) & =C(g(2,2))=\frac{(a-c)^{2}}{18} \\
W(g(1,1) & =W(g(2,2))=\frac{5(a-c)^{2}}{18}-4 k
\end{aligned}
$$

c) The distribution network $g(1,2)$ is symmetric to $g(2,1)$.

$$
\begin{aligned}
\Pi_{M_{1}}(g(1,2)) & =\Pi_{M_{2}}(g(1,2))=\Pi_{M_{1}}(g(2,1))=\Pi_{M_{2}}(g(2,1)) \\
& =\frac{2(2-d)(a-c)^{2}}{(2+d)(4-d)^{2}}-k \\
\Pi_{R_{1}}(g(1,2)) & =\Pi_{R_{2}}(g(1,2))=\Pi_{R_{1}}(g(2,1))=\Pi_{R_{2}}(g(2,1)) \\
& =\frac{4(a-c)^{2}}{(2+d)^{2}(4-d)^{2}}-k \\
\Phi(g(1,2)) & =\Phi(g(2,1))=\frac{4\left(6-d^{2}\right)(a-c)^{2}}{(2+d)^{2}(4-d)^{2}}-4 k \\
C(g(1,2) & =C(g(2,1))=\frac{4(a-c)^{2}}{(2+d)^{2}(4-d)^{2}} \\
W(g(1,2) & =W(g(2,1))=\frac{4\left(7-d^{2}\right)(a-c)^{2}}{(2+d)^{2}(4-d)^{2}}-4 k
\end{aligned}
$$

d) The distribution network $g(12,0)$ is symmetric to $g(0,12)$.

$$
\begin{aligned}
\Pi_{M_{1}}(g(12,0)) & =\Pi_{M_{2}}(g(12,0))=\Pi_{M_{1}}(g(0,12))=\Pi_{M_{2}}(g(0,12)) \\
& =\frac{(1-d)(a-c)^{2}}{2(1+d)(2-d)^{2}}-k \\
\Pi_{R_{1}}(g(12,0)) & =\Pi_{R_{2}}(g(0,12))=\frac{(a-c)^{2}}{2(1+d)(2-d)^{2}}-2 k \\
\Phi(g(12,0)) & =\Phi(g(0,12))=\frac{(3-2 d)(a-c)^{2}}{2(1+d)(2-d)^{2}}-4 k \\
C(g(12,0)) & =C(g(0,12))=\frac{2(a-c)^{2}}{8(1+d)^{2}(2-d)^{2}} \\
W(g(12,0)) & =W(g(0,12))=\frac{\left(7+2 d-4 d^{2}\right)(a-c)^{2}}{4(1+d)^{2}(2-d)^{2}}-4 k
\end{aligned}
$$


e) The distribution network $g(12,1)$ is symmetric to $g(12,2), g(1,12)$ and $g(2,12)$.

$$
\begin{aligned}
& \Pi_{M_{1}}(g(12,1))=\Pi_{M_{1}}(g(1,12))=\Pi_{M_{2}}(g(12,2))=\Pi_{M_{2}}(g(2,12)) \\
& =\frac{(1-d)(2-d)(2+d)(8+5 d)^{2}(a-c)^{2}}{6(1+d)\left(16-7 d^{2}\right)^{2}}-2 k \\
& \Pi_{M_{2}}(g(12,1))=\Pi_{M_{2}}(g(1,12))=\Pi_{M_{1}}(g(12,2))=\Pi_{M_{1}}(g(2,12)) \\
& =\frac{(1-d)\left(8+4 d-d^{2}\right)^{2}(a-c)^{2}}{2(1+d)\left(16-7 d^{2}\right)^{2}}-k \\
& \Pi_{R_{1}}(g(12,1))=\Pi_{R_{1}}(g(12,2))=\Pi_{R_{2}}(g(1,12))=\Pi_{R_{2}}(g(2,12)) \\
& =\frac{\left(52+28 d-7 d^{2}-d^{3}\right)(a-c)^{2}}{36(1+d)\left(16-7 d^{2}\right)}-2 k \\
& \Pi_{R_{2}}(g(12,1))=\Pi_{R_{2}}(g(12,2))=\Pi_{R_{1}}(g(1,12))=\Pi_{R_{1}}(g(2,12)) \\
& =\frac{\left(8+3 d-2 d^{2}\right)^{2}(a-c)^{2}}{9\left(16-7 d^{2}\right)^{2}}-k \\
& \Phi(g(12,1))=\Phi(g(12,2))=\Phi(g(1,12))=\Phi(g(2,12)) \\
& =\frac{(a-c)^{2}}{36(1+d)\left(16-7 d^{2}\right)^{2}}\left(3776+1280 d-3232 d^{2}\right. \\
& \left.-1192 d^{3}+509 d^{4}+155 d^{5}\right)-6 k \\
& C(g(12,1))=C(g(12,2))=C(g(1,12))=C(g(2,12)) \\
& =\frac{(a-c)^{2}}{72(1+d)^{2}\left(16-7 d^{2}\right)^{2}}\left(1600+1856 d-112 d^{2}\right. \\
& \left.-712 d^{3}-127 d^{4}+80 d^{5}+25 d^{6}\right) \\
& W(g(12,1))=W(g(12,2))=W(g(1,12))=W(g(2,12)) \\
& =\frac{(a-c)^{2}}{72(1+d)^{2}\left(16-7 d^{2}\right)^{2}}\left(9152+11968 d-4016 d^{2}\right. \\
& \left.-9560 d^{3}-1493 d^{4}+1408 d^{5}+335 d^{6}\right)-6 k
\end{aligned}
$$

\section{B Several results and proofs}

\section{B.1 Bounds on $k$}

\section{Proof of Lemma 1}

Consider first the distribution networks $g(1,0)$ and $g(1,1)$. From direct inspection of the agents' profits, the most binding constraint for $k$ is the one which ensures that $\Pi_{R_{1}}(g(1,1))>0$, that is, $k<\frac{(a-c)^{2}}{36}$. Similarly, for $g(1,2)$ the most binding constraint for $k$ is the one that imposes $\Pi_{R_{1}}(g(1,2))>0$, that is $k<\frac{4(a-c)^{2}}{(4-d)^{2}(2+d)^{2}}$. For $g(12,0)$, 
$\Pi_{R_{1}}(g(12,0))>0$ or equivalently $k<\frac{(a-c)^{2}}{4(2-d)^{2}(1+d)}$ is the most binding constraint. Comparing $\frac{(a-c)^{2}}{36}$ with $\frac{4(a-c)^{2}}{(4-d)^{2}(2+d)^{2}}$ and with $\frac{(a-c)^{2}}{4(2-d)^{2}(1+d)}$, it follows that the former expression is smaller than each of the other two expressions for $1>d$. For $g(12,1)$ and $g(12,12)$, it follows that $k<\frac{(a-c)^{2}}{36}$ is a more binding condition for $k$ than those imposed by $\Pi_{R_{1}}(g(12,1))>0, \Pi_{R_{2}}(g(12,1))>0$ and $\Pi_{R_{1}}(g(12,12))>0$ for $1>d$. Similarly, the condition on $k$ that ensures $\Pi_{M_{1}}(g(12,12))>0$, that is $k<\frac{(1-d)(a-c)^{2}}{3(2-d)^{2}(1+d)}$, is more binding that those ensuring $\Pi_{M_{1}}(g(12,1))>0$ and $\Pi_{M_{2}}(g(12,1))>0$. Therefore, by comparing expressions $\frac{(a-c)^{2}}{36}$ and $\frac{(1-d)(a-c)^{2}}{3(2-d)^{2}(1+d)}$ we have the upper bound on $k$ as a function of $d$ presented in Lemma 1.

\section{B.1.1 Pairwise stable distribution networks.}

\section{Proof of Proposition 1}

Remember that the link cost parameter is bounded above as indicated by Lemma 1 . We proceed by steps.

a) First, the distribution networks where one manufacturer and one retailer are out of the market (i.e. $g(1,0), g(2,0), g(0,1)$ and $g(0,2))$ are not pairwise stable since the manufacturer present in the market and the retailer selling no product would have incentives to create a link between them (i.e., $g(1,0)$ is defeated by $g(1,1)$ ).

b) Second, in case of distribution networks $g(1,1)$ and $g(2,2)$ there is one manufacturer out of the market and the other with two links. Note that no retailer wants to break its unique link. Furthermore, the manufacturer with two links wants to break one link if and only if $k>\frac{(a-c)^{2}}{24}$ which contradicts the restriction on $k<$ $\bar{k} \equiv \min \left\{\frac{(a-c)^{2}}{36}, \frac{(1-d)(a-c)^{2}}{3(1+d)(2-d)^{2}}\right\}$. Then, it remains to check whether any retailer wants to create a link with the manufacturer out of the market. Take for example $R_{1}$. $\Pi_{R_{1}}(g(12,1))=\frac{\left(52+28 d-7 d^{2}-d^{3}\right)(a-c)^{2}}{36(1+d)\left(16-7 d^{2}\right)}-2 k>\Pi_{R_{1}}(g(1,1))=\frac{(a-c)^{2}}{36}-k$ if and only if $\frac{\left(6+2 d+d^{3}\right)(a-c)^{2}}{6(1+d)\left(16-7 d^{2}\right)}>k$, which always holds since $\frac{\left(6+2 d+d^{3}\right)(a-c)^{2}}{6(1+d)\left(16-7 d^{2}\right)}>\frac{(1-d)(a-c)^{2}}{3(1+d)(2-d)^{2}}$. Then, we conclude that $g(1,1)$ and $g(2,2)$ are not pairwise stable since one link between a retailer and the manufacturer out of the market will always be created.

c) Third, in case of distribution networks $g(12,0)$ and $g(0,12)$ there is one retailer out of the market and the other with two links. No manufacturer wants to break its unique link. Furthermore, the retailer with two links wants to break one link if and only if $k>\frac{\left(4+3 d^{2}-d^{3}\right)(a-c)^{2}}{16(1+d)(2-d)^{2}}$. Since this expression is greater than $\bar{k}$ this retailer never breaks one link. Now we show that one manufacturer wants to create a link with the retailer out of the market. Take for example $M_{1} \cdot \Pi_{M_{1}}(g(12,1))=$ 
$\frac{(1-d)(2-d)(2+d)(8+5 d)^{2}(a-c)^{2}}{6(1+d)\left(16-7 d^{2}\right)^{2}}-2 k>\Pi_{M_{1}}(g(12,0))=\frac{(1-d)(a-c)^{2}}{2(1+d)(2-d)^{2}}-k$ if and only if $\frac{(1-d)\left(256-208 d^{2}+64 d^{3}+45 d^{4}-25 d^{5}\right)(a-c)^{2}}{6(2-d)^{2}\left(16-7 d^{2}\right)^{2}}>k$. This inequality always holds since the left hand side of the inequality is greater than $\bar{k}$. We conclude that the distribution networks $g(12,0)$ and $g(0,12)$ are not pairwise stable since one link between a manufacturer and the retailer out of the market will always be created.

d) Fourth, consider the distribution networks $g(1,2)$ and $g(2,1)$. First, note that no agent wants two break a link. Therefore we have to find the conditions under which both one manufacturer and one retailer want to create a link.

A manufacturer, say $M_{2}$, wants to create a link with a retailer, say $R_{1}$ if and only if $\Pi_{M_{2}}(g(12,2))=\frac{(1-d)(2-d)(2+d)(8+5 d)^{2}(a-c)^{2}}{6(1+d)\left(16-7 d^{2}\right)^{2}}-2 k>\Pi_{M_{2}}(g(1,2))=\frac{2(2-d)(a-c)^{2}}{(2+d)(4-d)^{2}}-k$ which is equivalent to $k<\frac{(2-d)\left(1024-1088 d^{2}-1120 d^{3}-728 d^{4}-52 d^{5}+45 d^{6}-25 d^{7}\right)(a-c)^{2}}{6(1+d)(2+d)(4-d)^{2}\left(16-7 d^{2}\right)^{2}}$ $\equiv k_{(1,2)}^{M}$. It is easy to check that $k_{(1,2)}^{M}>\bar{k}$ for $d \in(0,0.265)$ and is negative for $d \in(0.682,1)$.

Similarly, $R_{1}$ wants to create a link with $M_{2}$ if and only if $\Pi_{R_{1}}(g(12,2))=\frac{\left(52+28 d-7 d^{2}-d^{3}\right)(a-c)^{2}}{36(1+d)\left(16-7 d^{2}\right)}-2 k>\Pi_{R_{1}}(g(1,2))=\frac{4(a-c)^{2}}{(2+d)^{2}(4-d)^{2}}-k$ which is equivalent to $k<\frac{\left(1024+1152 d+832 d^{2}+176 d^{3}-8 d^{4}+68 d^{5}-3 d^{6}-d^{7}\right)(a-c)^{2}}{36(1+d)\left(16-7 d^{2}\right)(2+d)^{2}(4-d)^{2}} \equiv k_{(1,2)}^{R}$, where $k_{(1,2)}^{R}>\bar{k}$ for $d \in(0.295,1)$.

d.i) Thus, the distribution network $g(1,2)$ and $g(2,1)$ are stable if and only if $k>$ $\min \left\{k_{(1,2)}^{M}, k_{(1,2)}^{R}\right\}$, where $k_{(1,2)}^{R}<k_{(1,2)}^{M}$ if $d \in(0,0.269)$.

e) Fifth, consider distribution network $g(12,12)$. Note that the only way to break the stability of this distribution network is by breaking a link.

A manufacturer, say $M_{1}$, wants to break a link if and only if $\Pi_{M_{1}}(g(12,2))=$ $\frac{(1-d)\left(8+4 d-d^{2}\right)^{2}(a-c)^{2}}{2(1+d)\left(16-7 d^{2}\right)^{2}}-k>\Pi_{M_{1}}(g(12,12))=\frac{2(1-d)(a-c)^{2}}{3(1+d)(2-d)^{2}}-2 k$ which is equivalent to $k>\frac{(1-d)\left(256-320 d^{2}-96 d^{3}+88 d^{4}+36 d^{5}-3 d^{6}\right)(a-c)^{2}}{6(1+d)(2+d)^{2}\left(16-7 d^{2}\right)^{2}} \equiv k_{(12,12)}^{M}$, where $k_{(12,12)}^{M}>\bar{k}$ for $d \in(0,0.344)$ and is negative for $d \in(0.909,1)$.

Similarly, a retailer, say $R_{1}$ wants to break a link if and only if $\Pi_{R_{1}}(g(2,12))=$ $\frac{\left(8+3 d-2 d^{2}\right)^{2}(a-c)^{2}}{9\left(16-7 d^{2}\right)^{2}}-k>\Pi_{R_{1}}(g(12,12))=\frac{2(a-c)^{2}}{9(1+d)(2-d)^{2}}-2 k$ which is equivalent to $k>\frac{(1-d)\left(256+64 d-100 d^{2}+28 d^{3}-7 d^{4}-20 d^{5}+4 d^{6}\right)(a-c)^{2}}{9(1+d)(2+d)^{2}\left(16-7 d^{2}\right)} \equiv k_{(12,12)}^{R}$, where $0<k_{(12,12)}^{R}<\bar{k}$ for all $d \in(0,1)$.

e.i) The distribution network $g(12,12)$ is pairwise stable if and only if $k<\min \left\{k_{(12,12)}^{M}\right.$, $\left.k_{(12,12)}^{R}\right\}$, where $k_{(12,12)}^{R}<k_{(12,12)}^{M}$ if $d \in(0,0.480)$.

f) Finally, consider the distribution networks $g(12,1), g(12,2), g(1,12)$ and $g(2,12)$. Take for example $g(12,1)$. In this case $M_{2}$ and $R_{2}$ never want to break its unique link. $M_{1}$ does not want to break the link with $R_{2}$ since it is proved in $\mathbf{c}$ ) above that there is always an incentive to create a link between both. Similarly, $R_{1}$ does not want 
to break the link with $M_{2}$ since it is proved in $\mathbf{b}$ ) above that there is always an incentive to create a link between both. Considering the link between $M_{1}$ and $R_{1}$ it happens that $M_{1}$ will break the link if and only if $k>k_{(1,2)}^{M}$ while $R_{1}$ will do it if and only if $k>k_{(1,2)}^{R}$ (see $\mathbf{d}$ ) above). Finally, $M_{2}$ and $R_{2}$ will create a link if both have incentives to do it, that is if and only if $k<\min \left\{k_{(12,12)}^{M}, k_{(12,12)}^{R}\right\}$.

f.i) Thus, the distribution network $g(12,1)$ (and $g(12,2), g(1,12)$ and $g(2,12)$ ) is pairwise stable if and only if $k>\min \left\{k_{(12,12)}^{M}, k_{(12,12)}^{R}\right\}$ and $k<\min \left\{k_{(1,2)}^{M}, k_{(1,2)}^{R}\right\}$. Combining d.i), e.i) and f.i) yields the proposition.

\section{B.1.2 Strong stable distribution networks}

\section{Proof of Proposition 2}

First we show that $g(1,2)$ is always strongly stable when it is pairwise stable. In order for $g(1,2)$ not to be strongly stable with respect to $g(12,12)$, the coalition formed by the two manufacturers and the two retailers must benefit from the move. The four agents are better off with $g(12,12)$ if and only if $k<\min \left\{\frac{4\left(4-10 d+6 d^{2}-4 d^{3}+d^{4}\right)(a-c)^{2}}{3(1+d)(2+d)\left(8-6 d+d^{2}\right)}\right.$, $\left.\frac{-2\left(4-20 d+d^{2}\right)\left(2+2 d-d^{2}\right)(a-c)^{2}}{9(1+d)(2+d)^{2}\left(8-6 d+d^{2}\right)^{2}}\right\}$. The first term of the above expression corresponds to the threshold for $k$ denoted by $k_{s(1,2)}^{M}$, and such that for $k \leq k_{s(1,2)}^{M}$ the manufacturers want to create two links and move to $g(12,12)$. This threshold is decreasing with $d$ and reaches zero at $d=0.510$. The second term above is the threshold for $k$ denoted by $k_{s(1,2)}^{R}$, and such that for $k \leq k_{s(1,2)}^{R}$ retailers want to create two links and move to $g(12,12)$. This threshold increases with $d$ reaching zero at $d=0.202$. However, it is easy to check that the region defined by $0 \leq k \leq \min \left\{k_{s(1,2)}^{M}, k_{s(1,2)}^{R}\right\}$ does not intersect the region where $g(1,2)$ is pairwise stable (see Figure 4 ). In order for $g(1,2)$ not to be strongly stable with respect to $g(12,0)$, each member of the coalition $S=\left\{M_{2}, R_{1}\right\}$ must gain with the move that entails severing the link between $M_{2}$ and $R_{2}$ and creating a new link between them. However, it is easily proved that $M_{2}$ always prefers $g(1,2)$ since $\frac{2(2-d)(a-c)^{2}}{(4-d)^{2}(2+d)}$ is always greater than $\frac{(1-d)(a-c)^{2}}{2(2-d)^{2}(1+d)}$, and then $g(1,2)$ is strongly stable with respect to $g(12,0)$. In order for $g(1,2)$ not to be strongly stable with respect to $g(1,1)$, each member of the coalition $S=\left\{M_{1}, R_{2}\right\}$ must gain with the move that entails severing the link between $M_{2}$ and $R_{2}$ and creating a new link between them. However, it is easily proved that $R_{2}$ always prefers $g(1,2)$ since $\frac{4(a-c)^{2}}{(4-d)^{2}(2+d)^{2}}$ is always greater than $\frac{(a-c)^{2}}{36}$, and then $g(1,2)$ is strongly stable with respect to $g(1,1)$.

Considering the strong stability of $g(12,12)$ with respect to $g(1,2)$ it is enough that two agents decide to break a link each (the coalition $S$ that deviates is any coalition of cardinality two). Therefore, we conclude that $g(12,12)$ is not strongly stable for $k>$ 
$\min \left\{k_{s(1,2)}^{M}, k_{s(1,2)}^{R}\right\}$. To see if $g(12,12)$ is strongly stable we have to check if the coalition $S=\left\{M_{1}, M_{2}\right\}$ wants to move to $g(12,0)$. This happens for $k$ greater than $\frac{(1-d)(a-c)^{2}}{6(2-d)^{2}(1+d)}$. This later threshold is always greater than $k_{(12,12)}^{M}$ (see Figure 3), which means that the intersection between the region where $g(12,12)$ is pairwise stable and $k>\frac{(1-d)(a-c)^{2}}{6(2-d)^{2}(1+d)}$ is empty. Finally, we check the strong stability of $g(12,12)$ with respect to $g(1,1)$. The coalition to be considered is $S=\left\{R_{1}, R_{2}\right\}$ which has to break the links with $M_{2}$. Retailers gain in the move from $g(12,12)$ to $g(1,1)$ if and only if $k>\frac{\left(4+3 d^{2}-d^{3}\right)(a-c)^{2}}{36(2-d)^{2}(1-d)}$. But this inequality is never satisfied since $\bar{k}<\frac{\left(4+3 d^{2}-d^{3}\right)(a-c)^{2}}{36(2-d)^{2}(1-d)}$. Thus, $g(12,12)$ is strongly stable against $g(1,1)$. Summarizing, $g(12,12)$ is strongly stable for $k \leq \min \left\{k_{s(1,2)}^{M}, k_{s(1,2)}^{R}\right\}$.

Considering the strong stability of $g(12,1)$ with respect to $g(1,0)$, the coalition to be considered is $S=\left\{M_{1}, R_{1}\right\}$. Each agent in the coalition has to sever simultaneously its link with $R_{2}$ and $M_{2}$, respectively. It is easy to show that $M_{1}$ prefers to sever the link with $R_{2}$ if $k$ is greater than $\frac{\left(256-512 d-464 d^{2}+208 d^{3}+73 d^{4}-47 d^{5}\right)(a-c)^{2}}{24\left(16-7 d^{2}\right)^{2}(1+d)}$. Let us denote the later threshold by $k_{s(12,1)}^{M}$. This threshold is decreasing with $d$ and reaches zero at $d=0.3892$. Similarly, $R_{1}$ prefers to sever the link with $M_{2}$ if $k$ is greater than $\frac{\left(63-32 d+35 d^{2}+59 d^{3}\right)(a-c)^{2}}{144\left(16-7 d^{2}\right)(1+d)}$. We denote the later threshold by $k_{s(12,1)}^{R}$. It is always positive, initially decreasing with $d$ and then increasing reaching $\bar{k}$ at $d=0.7490$. We conclude that both agents in $S$ $=\left\{M_{1}, R_{1}\right\}$ gain with the move to $g(1,0)$ if $k>\max \left\{k_{s(12,1)}^{M}, k_{s(12,1)}^{R}\right\}$. Remind that $g(12,1)$ is pairwise stable in the region defined by the intersection of the $k$ satisfying $k<\min \left\{k_{(1,2)}^{M}, k_{(1,2)}^{R}\right\}$ and those satisfying $k>k_{(12,12)}^{R}$ (see Figure 3). Figure 4 displays the relationship between the thresholds under which $g(12,1)$ is pairwise stable and those that imply that the agents in the coalition $S=\left\{M_{1}, R_{1}\right\}$ gain with the move to $g(1,0)$. We therefore conclude that $g(12,1)$ is strongly stable in the region defined by the intersection of $k<\min \left\{k_{(1,2)}^{R}, k_{s(12,1)}^{M}\right\}$ and $k>k_{(12,12)}^{R}$.

\section{Characterization of closed cycles when no strongly stable network exists}

We should consider two different cases.

1. When $\min \left\{\min \left\{k_{(12,12)}^{R}, k_{(1,2)}^{M}\right\}, \max \left\{k_{s(12,1)}^{M}, k_{s(12,1)}^{R}\right\}\right\}>k>\min \left\{k_{s(1,2)}^{M}, k_{s(1,2)}^{R}\right\}$. In this area there is no strongly stable network and we observe a closed cycle formed by the networks $g(12,12), g(1,2), g(2,1), g(12,1), g(12,2), g(1,12)$ and $g(2,12)$. For such values of the link costs we know that the network $g(12,12)$ is not strongly stable because the coalition of two manufacturers, or the coalition of two retailers, or both coalitions, would have incentives to break two links moving to $g(1,2)$ (or $g(2,1))$. Once $g(1,2)$ (or $g(2,1))$ has been reached, we know by the proofs of pairwise and strong stability that no coalition has incentives to move to networks $g(1,0)$ (or $g(2,0), g(0,1), g(0,2)), g(1,1)$ (or $g(2,2))$, or $g(12,0)$ (or $g(0,12))$. However, the 
coalition formed by a manufacturer and a retailer would have incentives to form a link between them moving to $g(12,2)$ (or $g(12,1), g(1,12), g(2,12)$ ). Then, from $g(12,2)$, the coalition $S=\left\{M_{1}, R_{2}\right\}$ would like to form the link between them moving to $g(12,12)$. From $g(12,2)$ no coalition wants to move to any other network structure.

2. When $\min \left\{k_{(1,2)}^{R}, k_{(1,2)}^{M}\right\}>k>\max \left\{k_{s(12,1)}^{M}, k_{s(12,1)}^{R}\right\}$. In this area there is no strongly stable network and we observe a closed cycle formed by all possible networks; i.e., there exists an improving path connecting any two network structures. For such values of the link costs we know that neither the network $g(12,12)$ nor the network $g(12,1)$ (or $g(12,2), g(1,12)$, and $g(2,12)$ ) is strongly stable.

For $k<k_{(12,12)}^{R}, g(12,12)$ is not strongly stable because the coalition of two manufacturers, or the coalition of two retailers, or both coalitions, would have incentives to break two links moving to $g(1,2)$ (or $g(2,1))$. Once $g(1,2)$ (or $g(2,1)$ ) has been reached, the coalition formed by a manufacturer and a retailer would have incentives to form a link between them moving to $g(12,2)$ (or $g(12,1), g(1,12), g(2,12)$ ). From $g(12,2)$, the coalition $S=\left\{M_{1}, R_{2}\right\}$ would like to form the link between them moving to $g(12,12)$, but also coalition $S=\left\{M_{2}, R_{1}\right\}$ would like to sever simultaneously its link with $R_{2}$ and $M_{1}$ moving to $g(2,0)$. Once $g(2,0)$ has been reached, the networks $g(2,2), g(12,0)$ and $g(2,1)$ defeat the network $g(2,0)$. Next, the networks $g(2,2), g(12,0)$ and $g(2,1)$ are defeated by one of the asymmetric networks $g(12,2)$, $g(12,1), g(1,12)$, or $g(2,12)$. And so on.

For $k>k_{(12,12)}^{R}, g(12,1)$ (or $g(12,2), g(1,12)$, and $g(2,12)$ ) is not strongly stable because the coalition $S=\left\{M_{1}, R_{1}\right\}$ would like to sever simultaneously its link with $R_{2}$ and $M_{2}$ moving to $g(1,0)$. Once $g(1,0)$ has been reached, the networks $g(1,1)$, $g(12,0)$ and $g(1,2)$ defeat the network $g(1,0)$. Next, the networks $g(1,1), g(12,0)$ and $g(1,2)$ are defeated by one of the asymmetric networks $g(12,2), g(12,1), g(1,12)$, or $g(2,12)$. But the networks $g(1,1)$ and $g(12,0)$ are also defeated by $g(12,12)$, and $g(12,12)$ is defeated by any of the asymmetric networks $g(12,2), g(12,1), g(1,12)$, or $g(2,12)$. And so on.

\section{B.1.3 Efficient distribution networks}

We first fully characterize the efficient distribution networks. See Figure 6 .

Proposition 7 The efficient distribution network with positive link costs depends on both $d$ and $k$ as follows

a) For $0<d \leq 0.2156$ and 

a.1) $0<k<k^{B}(d)<\frac{(a-c)^{2}}{36}$ is $g(12,12)$
a.2) $0<k^{B}(d)<k<\frac{(a-c)^{2}}{36}$ is $g(1,2)$

b) For $0.2156<d \leq \frac{2}{3}$ and
b.1) $0<k<k^{A}(d)<k^{C}(d)<\frac{(a-c)^{2}}{36}$ is $g(12,12)$
b.2) $0<k^{A}(d)<k<k^{C}(d)<\frac{(a-c)^{2}}{36}$ is $g(12,1)$
b.3) $0<k^{A}(d)<k^{C}(d)<k<\frac{(a-c)^{2}}{36}$ is $g(1,2)$

c) c) For $\frac{2}{3}<d \leq 0.735$ and
c.1) $0<k<k^{A}(d)<k^{E}(d)<\frac{(a-c)^{2}}{36}$ is $g(12,12)$
c.2) $0<k^{A}(d)<k<k^{E}(d)<\frac{(a-c)^{2}}{36}$ is $g(12,1)$
c.3) $0<k^{A}(d)<k^{E}(d)<k<\frac{(a-c)^{2}}{36}$ is $g(12,0)$

d) For $0.735<d \leq 0.863$ and
d.1) $0<k<k^{E}(d)<\min \left\{\frac{(a-c)^{2}}{36}, \frac{(1-d)(a-c)^{2}}{3(1+d)(2-d)^{2}}\right\}$ is $g(12,1)$
d.2) $0<k^{E}(d)<k<\min \left\{\frac{(a-c)^{2}}{36}, \frac{(1-d)(a-c)^{2}}{3(1+d)(2-d)^{2}}\right\}$ is $g(12,0)$

e) For $0.863<d<1$ and $0<k<\frac{(1-d)(a-c)^{2}}{3(1+d)(2-d)^{2}}$ is $g(12,0)$

\section{Proof of Proposition 7 and Proposition 4 (main text).}

First note that $\Phi(g(12,0))-\Phi(g(1,0))>0$ if $k<\frac{(a-c)^{2}\left(12-16 d+9 d^{2}-3 d^{3}\right)}{32(2-d)^{2}(1+d)}$ which is a condition on $k$ milder than $\bar{k} \equiv \min \left\{\frac{(a-c)^{2}}{36}, \frac{(1-d)(a-c)^{2}}{3(1+d)(2-d)^{2}}\right\}$ since the difference $\left(12-16 d+9 d^{2}-\right.$ $\left.3 d^{3}\right) \frac{(a-c)^{2}}{32(2-d)^{2}(1+d)}-\frac{(a-c)^{2}}{36}$ is always positive. Therefore $\Phi(g(12,0))>\Phi(g(1,0))$ for all $k \in[0, \bar{k}]$.

Second, we show that $\Phi(g(12,0))-\Phi(g(1,1))=\frac{\left(11-18 d+12 d^{2}-4 d^{3}\right)(a-c)^{2}}{18(1+d)(2-d)^{2}}>0$ for all $k$ since both distribution networks have the same number of links and $\left(11-18 d+12 d^{2}-4 d^{3}\right)>0$ for $1>d$.

Third, the difference $\Phi(g(12,0))-\Phi(g(1,2))=\frac{d(-2+3 d)\left(16-14 d-3 d^{2}+2 d^{3}\right)(a-c)^{2}}{2(1+d)(4-d)^{2}(2-d)^{2}(2+d)^{2}}$ is independent of $k$ and positive as long as $d>\frac{2}{3}$. Therefore, we will proceed by considering two cases:

case a) $0<d<\frac{2}{3}$ where $\Phi(g(1,2))$ dominates $\Phi(g(12,0))$ and then, the possible efficient distribution networks are either $g(1,2)$, or $g(12,1)$, or $g(12,12)$. 
case b) $\frac{2}{3}<d<1$ where $\Phi(g(12,0))$ dominates $\Phi(g(1,2))$ and the possible efficient distribution networks are either $g(12,0)$, or $g(12,1)$, or $g(12,12)$.

case a) $0<d<\frac{2}{3}$ and $0 \leq k<\frac{(a-c)^{2}}{36}$.

a.1) We compare $g(12,12)$ with $g(12,1)$. The distribution network $g(12,12)$ is more efficient than $g(12,1)$ if $\frac{(1-d)\left(1280-1024 d-1088 d^{2}+224 d^{3}-212 d^{4}+44 d^{5}+155 d^{6}\right)(a-c)^{2}}{36(1+d)(2-d)^{2}\left(16-7 d^{2}\right)^{2}}-2 k$ is positive, or equivalently when $\frac{(1-d)(a-c)^{2}}{72(1+d)(2-d)^{2}\left(16-7 d^{2}\right)^{2}}\left(1280-1024 d-1088 d^{2}+224 d^{3}-\right.$ $\left.212 d^{4}+44 d^{5}+155 d^{6}\right)>k$. We denote the left part of the previous expression by $k^{A}(d)$. It follows that $k^{A}(d)$ is positive for $0<d<0.735$ and negative for $0.735<d<$ 1. We also have that $0<k^{A}(d)<\frac{(a-c)^{2}}{36}$ for all $0<d<\frac{2}{3}$. Therefore, the following conclusion follows: (i) $\Phi(g(12,12))>\Phi(g(12,1))$ if and only if $0 \leq k<k^{A}(d)$ and (ii) $\Phi(g(12,1))>\Phi(g(12,12))$ if and only if $k^{A}(d)<k<\frac{(a-c)^{2}}{36}$. Thus, for the particular case, $k=0$ and $0<d<\frac{2}{3}$, we have $\Phi(g(12,12))>\Phi(g(12,1))$.

a.2) We compare $g(12,12)$ with $g(1,2)$. Proceeding in the same way as before we have that: (i) $\Phi(g(12,12))>\Phi(g(1,2))$ if and only if $0 \leq k<k^{B}(d)$ and (ii) $\Phi(g(1,2))>$ $\Phi(g(12,12))$ if and only if $k^{B}(d)<k<\frac{(a-c)^{2}}{36}$, where $k^{B}(d)=\left(20-22 d-4 d^{2}+\right.$ $\left.3 d^{3}\right) \frac{\left(2-d+2 d^{2}\right)(a-c)^{2}}{9(1+d)(4-d)^{2}(2-d)^{2}(2+d)^{2}}$. It follows that $k^{B}(d)$ is positive for $0<d<0.861$ and negative for $0.861<d<1$ and that $0<k^{B}(d)<\frac{(a-c)^{2}}{36}$ for all $0<d<\frac{2}{3}$. Thus, for the particular case, $k=0$ and $0<d<\frac{2}{3}$, it follows that $\Phi(g(12,12))>\Phi(g(1,2))$. Together with the above conclusion for $k=0$ implies that the efficient network is $g(12,12)$ for $0<d<\frac{2}{3}$.

a.3) We compare $g(12,1)$ with $g(1,2)$. We have that: (i) $\Phi(g(12,1))>\Phi(g(1,2))$ if and only if $0<k<k^{C}(d)$ and (ii) $\Phi(g(1,2))>\Phi(g(12,1))$ if and only if $k^{C}(d)<$ $k<\frac{(a-c)^{2}}{36}$, where $k^{C}(d)$ is equal to $\frac{(1-d)(a-c)^{2}}{72(1+d)(4-d)^{2}(2+d)^{2}\left(16-7 d^{2}\right)^{2}}(20480-18432 d+$ $\left.19200 d^{2}+20224 d^{3}-42720 d^{4}-19872 d^{5}+7444 d^{6}+1968 d^{7}-111 d^{8}+155 d^{9}\right)$. It follows that $k^{C}(d)$ is positive for $0<d<0.893$ and negative for $0.893<d<1$ and that $0<k^{C}(d)<\frac{(a-c)^{2}}{36}$ for all $0<d<\frac{2}{3}$.

The final step before proving the proposition is to compare the three thresholds $k^{A}(d), k^{B}(d)$, and $k^{C}(d)$ for $0<d<\frac{2}{3}$. It is easy to check that $k^{A}(d)>k^{B}(d)>$ $k^{C}(d)$ for $0<d<0.2156$ and $k^{A}(d)<k^{B}(d)<k^{C}(d)$ for $0.2156<d<\frac{2}{3}$. It also happens that for $d=0$ and $d=0.2156$ all of them are equal. Therefore, the efficient distribution network depends on the size of $k$ and the value of $d$ as follows:

case a.i) $0<d<0.2156$ and $0<k^{C}(d)<k^{B}(d)<k^{A}(d)<\frac{(a-c)^{2}}{36}$.

(1) If $0<k<k^{C}(d)<k^{B}(d)<k^{A}(d)$ then $\Phi(g(12,1))>\Phi(g(1,2)), \Phi(g(12,12))>$ 
$\Phi(g(1,2))$, and $\Phi(g(12,12))>\Phi(g(12,1))$. Therefore, $g(12,12)$ is the efficient distribution network.

(2) If $0<k^{C}(d)<k<k^{B}(d)<k^{A}(d)$ then $\Phi(g(12,1))<\Phi(g(1,2)), \Phi(g(12,12))>$ $\Phi(g(1,2))$, and $\Phi(g(12,12))>\Phi(g(12,1))$. As before, $g(12,12)$ is the efficient distribution network.

(3) If $0<k^{C}(d)<k^{B}(d)<k<k^{A}(d)$ then $\Phi(g(12,1))<\Phi(g(1,2)), \Phi(g(12,12))<$ $\Phi(g(1,2))$, and $\Phi(g(12,12))>\Phi(g(12,1))$. Therefore, $g(1,2)$ is the efficient distribution network.

(4) If $0<k^{C}(d)<k^{B}(d)<k^{A}(d)<k<\frac{(a-c)^{2}}{36}$ then $\Phi(g(12,1))<\Phi(g(1,2))$, $\Phi(g(12,12))<\Phi(g(1,2))$, and $\Phi(g(12,12))>\Phi(g(12,1))$. Hence, $g(1,2)$ is the efficient distribution network.

case a.ii) $0.2156<d<\frac{2}{3}$ and $0<k^{A}(d)<k^{B}(d)<k^{C}(d)<\frac{(a-c)^{2}}{36}$. Following the same reasoning as before we have that either, $g(12,12)$ is the efficient distribution network for $0<k<k^{A}(d)<k^{B}(d)<k^{C}(d)$, or $g(12,1)$ for $0<k^{A}(d)<k<k^{C}(d)$, or $g(1,2)$ for $0<k^{C}(d)<k<\frac{(a-c)^{2}}{36}$.

Figure 8 summarizes the above result. The area A corresponds to the area where $g(12,12)$ is the efficient distribution network, the area B is the one where $g(1,2)$ is efficient, and the uncolored area corresponds to the area where $g(12,1)$ is the efficient distribution network.

case b) $\frac{2}{3}<d<1$ and $0<k<\bar{k}$.

b.1) We compare $g(12,12)$ with $g(12,0))$. We conclude that: (i) $\Phi(g(12,12))>\Phi(g(12,0))$ if and only if $0<k<k^{D}(d)$ and (ii) $\Phi(g(12,0))>\Phi(g(12,12))$ if and only if $k^{D}(d)<k<\bar{k}$, where $k^{D}(d)=\frac{(5-6 d)(a-c)^{2}}{72(1+d)(2-d)^{2}}$. It follows that $k^{D}(d)$ is positive for $0<d<\frac{5}{6}$ and negative for $\frac{5}{6}<d<1$, and that $0<k^{D}(d)<\bar{k}$ for all $0<d<1$. Therefore, for $\frac{2}{3}<d<\frac{5}{6}$ (i) and (ii) are possible, while for $\frac{5}{6}<d<1$ only (ii) is possible.

b.2) We compare $g(12,1)$ with $g(12,0))$. We conclude that: (i) $\Phi(g(12,1))>\Phi(g(12,0))$ if and only if $0<k<k^{E}(d)$ and (ii) $\Phi(g(12,0))>\Phi(g(12,1))$ if and only if $k^{E}(d)<$ $k<\bar{k}$, where $k^{E}(d)=\frac{\left(1280-2048 d-128 d^{2}+1504 d^{3}-578 d^{4}-266 d^{5}+155 d^{6}\right)(a-c)^{2}}{72(2-d)^{2}\left(16-7 d^{2}\right)^{2}}$. It follows that $k^{E}(d)$ is positive for $0<d<0.863$ and negative for $0.863<d<1$ and that $0<k^{E}(d)<\bar{k}$ for all $0<d<1$. Then, for $\frac{2}{3}<d<0.863$ (i) and (ii) are possible, while for $0.863<d<1$ only (ii) is possible.

b.3) See a.1) for the comparison between $g(12,12)$ and $g(12,1)$. 


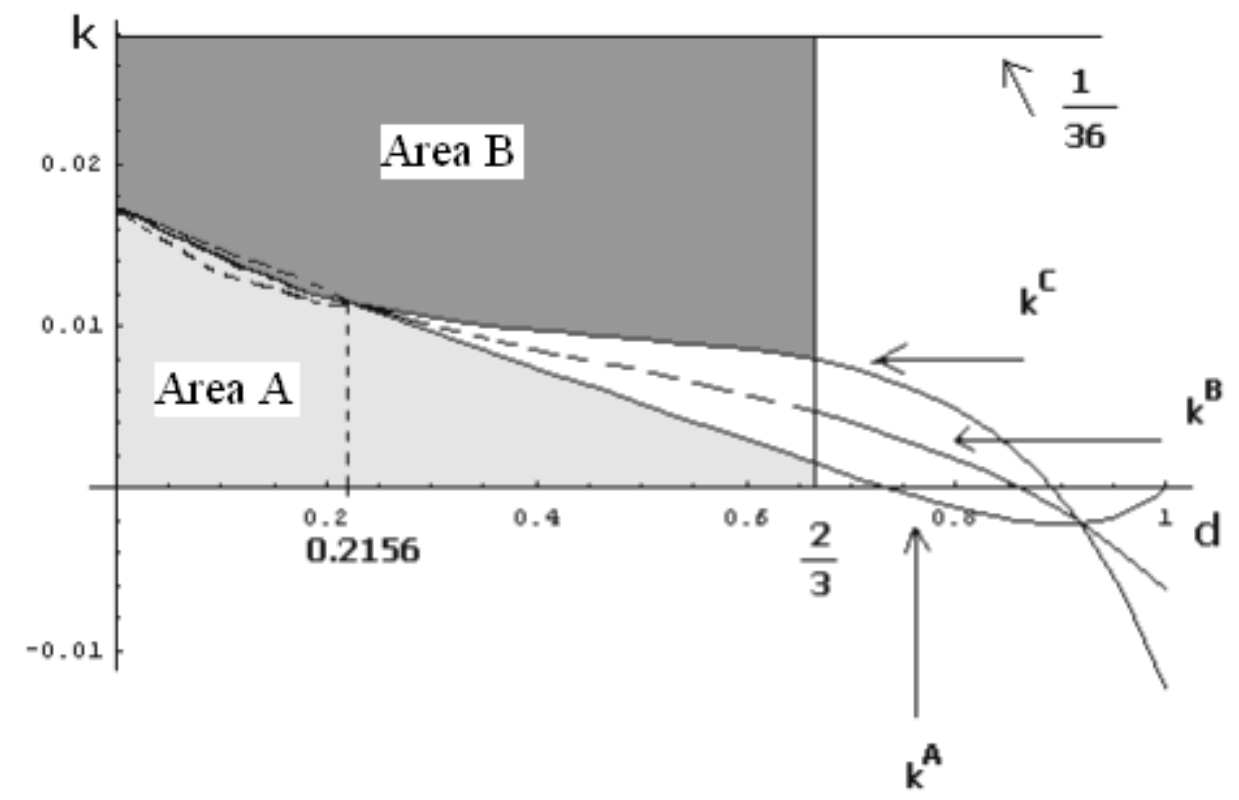

Figure 8: Efficient distribution networks when $0<d<\frac{2}{3}$.

Putting together b.1), b.2) and b.3) it follows that $k^{A}(d)<k^{D}(d)<k^{E}(d)<\bar{k}$. Four different cases can be distinguished:

(i) $0<k<k^{A}(d)<k^{D}(d)<k^{E}(d)<\bar{k}$, where $\Phi(g(12,12))>\Phi(g(12,1))$, $\Phi(g(12,12))>$ $\Phi(g(12,0))$ and $\Phi(g(12,1))>\Phi(g(12,0))$, with the conclusion that $g(12,12)$ is the efficient distribution network.

(ii) $k^{A}(d)<k<k^{D}(d)<k^{E}(d)<\bar{k}$, where $\Phi(g(12,12))<\Phi(g(12,1)), \Phi(g(12,12))>$ $\Phi(g(12,0))$ and $\Phi(g(12,1))>\Phi(g(12,0))$, with the conclusion that $g(12,1)$ is the efficient distribution network.

(iii) $k^{A}(d)<k^{D}(d)<k<k^{E}(d)<\bar{k}$, where $\Phi(g(12,12))<\Phi(g(12,1)), \Phi(g(12,12))<$ $\Phi(g(12,0))$ and $\Phi(g(12,1))>\Phi(g(12,0))$, with the conclusion that $g(12,1)$ is the efficient distribution network.

(iv) $k^{A}(d)<k^{D}(d)<k^{E}(d)<k<\bar{k}$, where $\Phi(g(12,12))<\Phi(g(12,1)), \Phi(g(12,12))<$ $\Phi(g(12,0))$ and $\Phi(g(12,1))<\Phi(g(12,0))$, with the conclusion that $g(12,0)$ is the efficient distribution network. 
Figure 9 displays the efficient distribution networks for case b) $\frac{2}{3}<d<1$. The area $\mathrm{B}$ corresponds to the area where $g(12,12)$ is efficient, the uncolored area corresponds to the area where $g(12,1)$ is efficient, and finally the area A corresponds to the area where $g(12,0)$ is efficient.

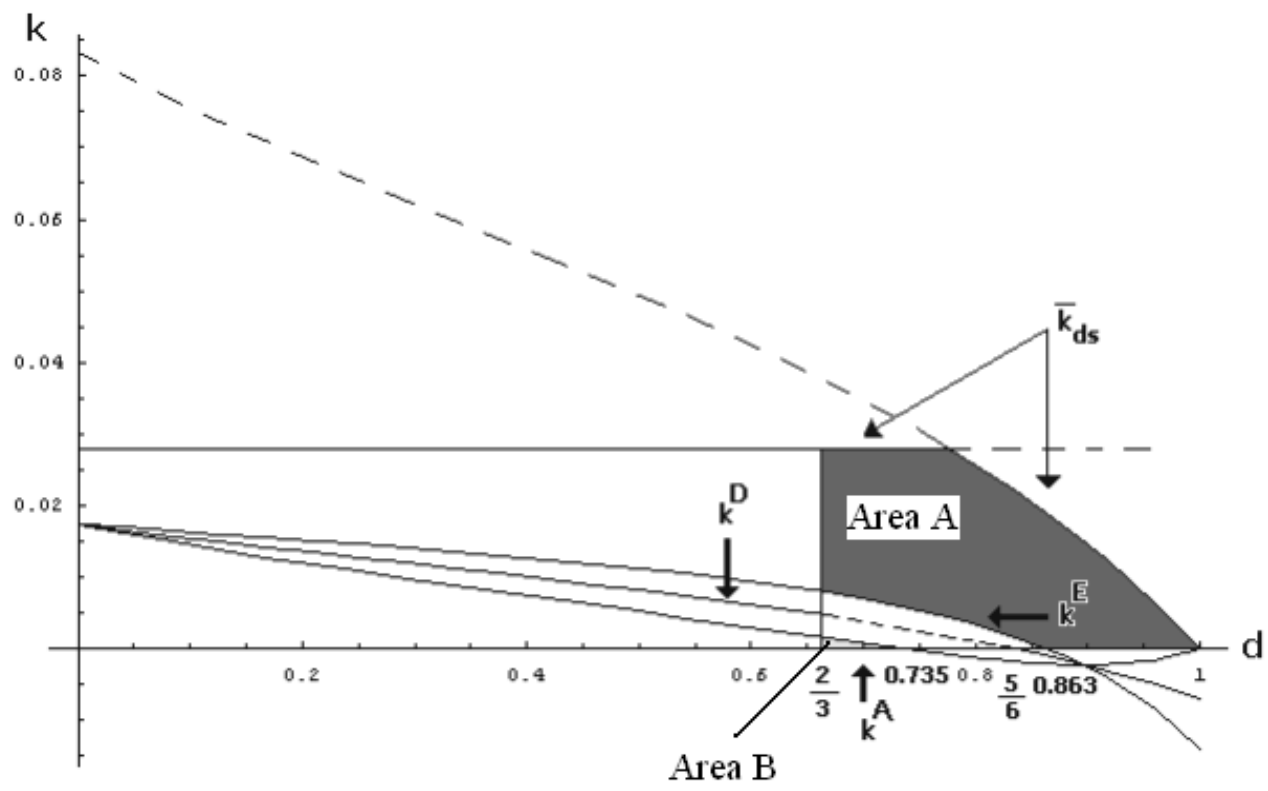

Figure 9: Efficient distribution networks when $\frac{2}{3}<d<1$.

As a corollary, for the particular case $k=0$ and $\frac{2}{3}<d<1$, we have that $g(12,12)$ is the efficient distribution network for $\frac{2}{3}<d<0.735$ since $0<k^{A}(d)<k^{D}(d)<k^{E}(d)<\bar{k}$ and (i) above applies; $g(12,1)$ is the efficient distribution network for $0.735<d<0.863$ since $k^{A}(d)<0<k^{D}(d)<k^{E}(d)<\bar{k}$ and (iii) above applies; finally $g(12,0)$ is the efficient distribution network for $0.863<d<1$ since $k^{E}(d)<0<\bar{k}$ and (iv) above applies.

\section{B.1.4 Consumer surplus analysis}

We give the complete characterization of Proposition 5 in the main text and its proof.

Proposition 8 The consumer surplus ranking is a function of the size of $d$ as follows.

a) $C(g(12,12))>C(g(12,1))>C(g(1,2))>C(g(12,0)) \geq C(g(1,1))>C(g(1,0))$ for $0<d \leq 0.1413$,

b) $C(g(12,12))>C(g(12,1))>C(g(1,2))>C(g(1,1)) \geq C(g(12,0))>C(g(1,0))$ for $0.1413<d \leq 0.2826$, 
c) $C(g(12,12))>C(g(12,1))>C(g(1,1))>C(g(1,2)) \geq C(g(12,0))>C(g(1,0))$ for $0.2826<d \leq \frac{2}{3}$,

d) $C(g(12,12))>C(g(12,1))>C(g(1,1))>C(g(12,0)) \geq C(g(1,2))>C(g(1,0))$ for $\frac{2}{3}<d \leq 0.8597$,

e) $C(g(12,12))>C(g(12,1))>C(g(12,0))>C(g(1,1))>C(g(1,2))>C(g(1,0))$ for $0.8597<d<1$.

Proof: All expressions of the consumer surplus corresponding to the different distribution networks are multiplied by the factor $(a-c)^{2}$. Then, the comparisons are independent of this factor and we will ignore it throughout this proof.

(i) $C(g(1,1))>C(g(1,0))$ if and only if $\frac{7}{288 b}>0$ which always holds.

(ii) $C(g(1,2))>C(g(1,0))$ if and only if $64-32 d+12 d^{2}+4 d^{3}-d^{4}>0$, which always holds since $1>d$.

(iii) $C(g(12,0))>C(g(1,0))$ if and only if $4-4 d+3 d^{2}+2 d^{3}-d^{4}>0$, which always holds since $1>d$.

(iv) $C(g(12,1))>C(g(1,1))$ if and only if $192-64 d-80 d^{2}+360 d^{3}+191 d^{4}-104 d^{5}-57 d^{6}>$ 0 , which always holds because $1>d$.

(v) $C(g(12,1))>C(g(12,0))$ if and only if $1792-768 d-1472 d^{2}+928 d^{3}+418 d^{4}-302 d^{5}-$ $45 d^{5}+25 d^{6}>0$, which always holds since $1>d$.

(vi) $C(g(12,1))>C(g(1,2))$ if and only if $28672+22528 d+23808 d^{2}+51200 d^{3}+15008 d^{4}-$ $16320 d^{5}-5692 d^{6}-364 d^{7}-747 d^{8}-20 d^{9}+25 d^{10}>0$, which always holds given that $1>d$.

(vii) $C(g(12,12))>C(g(12,1))$ if and only if $1792-1024 d-896 d^{2}+544 d^{3}-660 d^{4}-$ $116 d^{5}+347 d^{6}+20 d^{7}-25 d^{8}>0$, which always holds because $1>d$.

Using (i) to (vii) we have that $C(g(1,0))$ is last in the ranking, and $C(g(12,12))$ and $C(g(12,1))$ are first and second in the ranking, respectively. It remains to specify the ranking among $C(g(12,0)), C(g(1,1))$ and $C(g(1,2))$. It follows that $C(g(1,2))>C(g(1,1))$ if and only if $1-32 d+12 d^{2}+4 d^{3}-d^{4}>0$, that is for $0<d<0.2826$. Similarly, $C(g(1,2))>C(g(12,0))$ if and only if $0<d<\frac{2}{3}$. Finally, $C(g(12,0))>C(g(1,1))$ if and only if $1-8 d+6 d^{2}+4 d^{3}-2 d^{4}>0$, that is for both $0<d<0.1413$ and $0.8587<d<1$. Combining the three conditions above yields the proposition. For instance, for $0<d \leq 0.1413$, it follows that $C(g(1,2))>C(g(1,1)), C(g(1,2))>C(g(12,0))$ 
and $C(g(12,0))>C(g(1,1))$ yielding $\mathbf{a})$ in the proposition.

\section{B.1.5 Social welfare analysis}

We give the complete characterization of Proposition 6 in the main text and its proof.

Proposition 9 The distribution network that maximizes social welfare is

a) $g(12,12)$ when either
a.1) $0<d \leq 0.032569$ and $0 \leq k<\bar{k}$, or
a.2) $0.032569<d \leq 0.184824$ and $0 \leq k<k_{(12,12)-(1,2)}^{s w}$, or
a.3) $0.184824<d \leq 0.9535$ and $0 \leq k<k_{(12,12)-(12,1)}^{s w}$.

b) $g(12,1)$ when either
b.1) $0.184824<d \leq \frac{2}{3}$ and $k_{(12,12)-(12,1)}^{s w} \leq k<k_{(12,1)-(1,2)}^{s w}$, or
b.2) $\frac{2}{3}<d \leq 0.87953$ and $k_{(12,12)-(12,1)}^{s w} \leq k<k_{(12,1)-(12,0)}^{s w}$, or
b.3) $0.87953<d<0.9535$ and $k_{(12,12)-(12,1)}^{s w} \leq k<\bar{k}$, or
b.4) $0.9535<d<1$ and $0 \leq k<\bar{k}$.

c) $g(1,2)$ when either

$$
\begin{aligned}
& \text { c.1) } 0.032569<d \leq 0.184824 \text { and } k_{(12,12)-(1,2)}^{s w} \leq k<\bar{k} \text {, or } \\
& \text { c.2) } 0.184824<d \leq \frac{2}{3} \text { and } k_{(12,1)-(1,2)}^{s w} \leq k<\bar{k} \text {. }
\end{aligned}
$$

d) $g(12,0)$ when $\frac{2}{3}<d \leq 0.87953$ and $k_{(12,1)-(12,0)}^{s w} \leq k<\bar{k}$.

Proof: We first show that the distribution networks $g(1,0)$ and $g(1,1)$ are always dominated in social welfare terms by some other distribution network. First note that the difference $W(g(1,1))-W(g(1,0))=\frac{17(a-c)^{2}}{288}-2 k$ is positive for all $k \in[0, \bar{k})$ since $\frac{17(a-c)^{2}}{576}>\bar{k}$, and therefore $g(1,0)$ does not attain the highest social welfare. Second, $W(g(1,2))-W(g(1,1))=\frac{\left(184-160 d-12 d^{2}+20 d^{3}-5 d^{4}\right)(a-c)^{2}}{18(4-d)^{2}(2+d)^{2}}>0$ since $1>d>0$, and therefore the distribution network $g(1,1)$ does not maximize social welfare.

Next, the difference $W(g(1,2))-W(g(12,0))=\frac{d(2-3 d)\left(48+10 d-39 d^{2}-2 d^{3}-4 d^{4}\right)(a-c)^{2}}{4(4-d)^{2}(2-d)^{2}(2+d)^{2}(1+d)^{2}}$ is also independent of $k$ and positive as long as $0<d<\frac{2}{3}$. Then, $W(g(1,2))>W(g(12,0))$ for $0<d<\frac{2}{3}$; and the opposite otherwise. Thus, we will consider two cases: (a) for $0<d<\frac{2}{3}$ where only $g(1,2), g(12,1)$ and $g(12,12)$ have to be considered, and (b) for $\frac{2}{3}<d<1$ where the distribution networks to be analyzed are $g(12,0), g(12,1)$ and $g(12,12)$. 
(a) $0<d<\frac{2}{3}$.

We first define the thresholds on $k$ that indicate which one of the three distribution networks $g(1,2), g(12,1)$ and $g(12,12)$ is the one that achieves the greatest social welfare.

- The difference $W(g(12,1))-W(g(1,2))$ is positive if $k<(69632+26624 d+$ $25344 d^{2}+130048 d^{3}-29984 d^{4}-141504 d^{5}-30548 d^{6}+18460 d^{7}+2967 d^{8}+68 d^{9}+$ $\left.335 d^{10}\right) \frac{(a-c)^{2}}{144(4-d)^{2}(2+d)^{2}(1+d)^{2}\left(16-7 d^{2}\right)^{2}}$. Denote by $k_{(12,1)-(1,2)}^{s w}$ the later expression, which is a function of $d$, is always positive and intersects $\bar{k}$ in the interval $0<d<\frac{2}{3}$ at $d=0.0296709$, being $k_{(12,1)-(1,2)}^{s w}>\bar{k}$ for $0<d<0.0296709$ and the opposite for $0.0296709<d<\frac{2}{3}$.

- The difference $W(g(12,12))-W(g(1,2))$ is positive if $k<\left(34+3 d-30 d^{2}-d^{3}+\right.$ $\left.3 d^{4}\right) \frac{\left(2-d+d^{2}\right)(a-c)^{2}}{9(4-d)^{2}(2-d)^{2}(2+d)^{2}(1+d)^{2}}$. We denote by $k_{(12,12)-(1,2)}^{s w}$ the later expression, which is always positive and intersects $\bar{k}$ at $d=0.0325694$ in the interval $0<d<\frac{2}{3}$, being $k_{(12,12)-(1,2)}^{s w}>\bar{k}$ for $0<d<0.0325694$ and the opposite for $0.0325694<d<\frac{2}{3}$.

- The difference $W(g(12,12))-W(g(12,1))$ is positive if $k<\frac{(a-c)^{2}}{144(2-d)^{2}(1+d)^{2}\left(16-7 d^{2}\right)^{2}}$ $\left(4352-3072 d-5632 d^{2}+3040 d^{3}+1096 d^{4}-476 d^{5}+1081 d^{6}-68 d^{7}-335 d^{8}\right)$. We denote by $k_{(12,12)-(12,1)}^{s w}$ the later expression, which is positive for $0<d<0.953503$ and intersects $\bar{k}$ at $d=0.0359544$, being $k_{(12,12)-(12,1)}^{s w}>\bar{k}$ for $0<d<0.0359544$ and the opposite for $0.0359544<d<\frac{2}{3}$. Further note that at $d=0$ and at $d=184824$ the three thresholds coincide; for $0<d<0.184824$ they are ranked as $k_{(12,1)-(1,2)}^{s w}<k_{(12,12)-(1,2)}^{s w}<k_{(12,12)-(12,1)}^{s w}$, while for $0.184824<d<\frac{2}{3}$ the ranking is $k_{(12,12)-(12,1)}^{s w}<k_{(12,12)-(1,2)}^{s w}<k_{(12,1)-(1,2)}^{s w}$. Then, the following subcases can be distinguished:

(a.i) For $0<d<0.0296709$, it follows that $\bar{k}<k_{(12,1)-(1,2)}^{s w}<k_{(12,12)-(1,2)}^{s w}<k_{(12,12)-(12,1)}^{s w}$. Then, for all $k$ belonging to $[0, \bar{k}]$ the three differences defined above are positive and then $g(12,12)$ maximizes the social welfare.

(a.ii) For $0.0296709<d<0.032569$, it follows that $0<k_{(12,1)-(1,2)}^{s w}<\bar{k}<k_{(12,12)-(1,2)}^{s w}<$ $k_{(12,12)-(12,1)}^{s w}$. Then for all $k$ belonging to $[0, \bar{k}]$ we have that both $W(g(12,12))-$ $W(g(1,2))$ and $W(g(12,12))-W(g(12,1))$ are positive and then $W(g(12,12))$ is the greatest. Items (a.i) and (a.ii) together prove part a.1) of the proposition.

(a.iii) For $0.032569<d<0.035954$, it follows that $0<k_{(12,1)-(1,2)}^{s w}<k_{(12,12)-(1,2)}^{s w}<\bar{k}<$ $k_{(12,12)-(12,1)}^{s w}$. Then, for $0 \leq k<k_{(12,1)-(1,2)}^{s w}<k_{(12,12)-(1,2)}^{s w}<\bar{k}<k_{(12,12)-(12,1)}^{s w}$, or $0<k_{(12,1)-(1,2)}^{s w}<k<k_{(12,12)-(1,2)}^{s w}<\bar{k}<k_{(12,12)-(12,1)}^{s w}$, the following inequalities $W(g(12,12))>W(g(1,2))$ and $W(g(12,12))>W(g(12,1))$ are satisfied. Then part a.2) in the proposition is proved. But for $0<k_{(12,1)-(1,2)}^{s w}<k_{(12,12)-(1,2)}^{s w}<k<$ 
$\bar{k}<k_{(12,12)-(12,1)}^{s w}$, it follows that $W(g(12,12))<W(g(1,2))$ and $W(g(12,1))<$ $W(g(1,2))$. Then $W(g(1,2))$ gives the highest social welfare.

(a.iv) For $0.035954<d<0.184824$, it follows that $0<k_{(12,1)-(1,2)}^{s w}<k_{(12,12)-(1,2)}^{s w}<$ $k_{(12,12)-(12,1)}^{s w}<\bar{k}$. Then, for $0 \leq k<k_{(12,1)-(1,2)}^{s w}<k_{(12,12)-(1,2)}^{s w}<k_{(12,12)-(12,1)}^{s w}<\bar{k}$, or $0<k_{(12,1)-(1,2)}^{s w}<k<k_{(12,12)-(1,2)}^{s w}<k_{(12,12)-(12,1)}^{s w}<\bar{k}$ the greatest social welfare is achieved by $g(12,12)$; while for $0<k_{(12,1)-(1,2)}^{s w}<k_{(12,12)-(1,2)}^{s w}<k<$ $k_{(12,12)-(12,1)}^{s w}<\bar{k}$ or $0<k_{(12,1)-(1,2)}^{s w}<k_{(12,12)-(1,2)}^{s w}<k_{(12,12)-(12,1)}^{s w}<k<\bar{k}, g(1,2)$ gives the highest social welfare. Items (a.iii) and (a.iv) prove part c.1) of the proposition.

(a.v) For $0.184824<d<\frac{2}{3}$, we have $0<k_{(12,12)-(12,1)}^{s w}<k_{(12,12)-(1,2)}^{s w}<k_{(12,1)-(1,2)}^{s w}<\bar{k}$. Then, for $0 \leq k<k_{(12,12)-(12,1)}^{s w}<k_{(12,12)-(1,2)}^{s w}<k_{(12,1)-(1,2)}^{s w}<\bar{k}$ the greatest social welfare is attained with $g(12,12)$. For either $0<k_{(12,12)-(12,1)}^{s w}<k<k_{(12,12)-(1,2)}^{s w}<$ $k_{(12,1)-(1,2)}^{s w}<\bar{k}$ or $0<k_{(12,12)-(12,1)}^{s w}<k_{(12,12)-(1,2)}^{s w}<k<k_{(12,1)-(1,2)}^{s w}<\bar{k}, g(12,1)$ gives the highest social welfare. Finally, for $0<k_{(12,12)-(12,1)}^{s w}<k_{(12,12)-(1,2)}^{s w}<$ $k_{(12,1)-(1,2)}^{s w}<k<\bar{k}$ the greatest social welfare is $W(g(1,2))$. This proves parts b.1) and $\mathbf{c . 2}$ ) of the proposition.

(b) $\frac{2}{3}<d<1$.

We now define the thresholds on $k$ that indicate which one of $g(12,0), g(12,1)$ and $g(12,12)$ is the one that achieves the greatest social welfare.

- The difference $W(g(12,1))-W(g(12,0))$ is positive if $k<\left(4352-2304 d-5824 d^{2}+\right.$ $\left.3680 d^{3}+2270 d^{4}-1990 d^{5}-267 d^{6}+335 d^{7}\right) \frac{(a-c)^{2}}{144(2-d)^{2}(1+d)\left(16-7 d^{2}\right)^{2}}$. Denote by $k_{(12,1)-(12,0)}^{s w}$ the later expression, which is a function of $d$, is always positive and intersects $\bar{k}$ in the interval $\frac{2}{3}<d<1$ at $\frac{d}{b}=0.87953$. Then, for $\frac{2}{3}<d<0.87953$ it follows that $k_{(12,1)-(12,0)}^{s w}<\bar{k}$, the opposite follows for $d>0.87953$.

- The difference $W(g(12,12))-W(g(12,0))$ is positive if $k<\frac{\left(17-2 d+12 d^{2}\right)(a-c)^{2}}{144(2-d)^{2}(1+d)^{2}}$. We denote by $k_{(12,12)-(12,0)}^{s w}$ the later expression, which is always positive and intersects $\bar{k}$ at $d=0.956154$ in the interval $\frac{2}{3}<d<1$. Then, for $\frac{2}{3}<d<0.956154$ it follows that $k_{(12,12)-(12,0)}^{s w}<\bar{k}$, the opposite follows for $d>0.956154$.

- The threshold for the difference $W(g(12,12))-W(g(12,1))$ is the same as in case (a), $k_{(12,12)-(12,1)}^{s w}$. The following ranking for the thresholds applies in the interval $\frac{2}{3}<d<1: k_{(12,12)-(12,1)}^{s w}<k_{(12,12)-(12,0)}^{s w}<k_{(12,1)-(12,0)}^{s w}$. The following subcases are analyzed:

(b.i) For $\frac{2}{3}<d<0.87953$, we have $0<k_{(12,12)-(12,1)}^{s w}<k_{(12,12)-(12,0)}^{s w}<k_{(12,1)-(12,0)}^{s w}<\bar{k}$. Then, for $0 \leq k<k_{(12,12)-(12,1)}^{s w}<k_{(12,12)-(12,0)}^{s w}<k_{(12,1)-(12,0)}^{s w}<\bar{k}$ the greatest social welfare is obtained with $g(12,12)$. For either $0<k_{(12,12)-(12,1)}^{s w}<k<$ 
$k_{(12,12)-(12,0)}^{s w}<k_{(12,1)-(12,0)}^{s w}<\bar{k}$ or $0<k_{(12,12)-(12,1)}^{s w}<k_{(12,12)-(12,0)}^{s w}<k<$ $k_{(12,1)-(12,0)}^{s w}<\bar{k}, g(12,1)$ gives the highest social welfare, and part $\left.\mathbf{b} .2\right)$ of the proposition is proved. Finally, for $0<k_{(12,12)-(12,1)}^{s w}<k_{(12,12)-(12,0)}^{s w}<k_{(12,1)-(12,0)}^{s w}<k<$ $\bar{k}$ the greatest social welfare is $W(g(12,0))$, and part $\mathbf{d})$ of the proposition is proved.

(b.ii) For $0.87953<d<0.9535$, it follows that $0<k_{(12,12)-(12,1)}^{s w}<k_{(12,12)-(12,0)}^{s w}<\bar{k}<$ $k_{(12,1)-(12,0)}^{s w}$. Then, for $0 \leq k<k_{(12,12)-(12,1)}^{s w}<k_{(12,12)-(12,0)}^{s w}<\bar{k}<k_{(12,1)-(12,0)}^{s w}$ the greatest social welfare is obtained with $g(12,12)$. Considering items (a.v), (b.i) and (b.ii), part a.3) of the proposition is proved. For either $0<k_{(12,12)-(12,1)}^{s w}<$ $k<k_{(12,12)-(12,0)}^{s w}<\bar{k}<k_{(12,1)-(12,0)}^{s w}$ or $0<k_{(12,12)-(12,1)}^{s w}<k_{(12,12)-(12,0)}^{s w}<k<\bar{k}<$ $k_{(12,1)-(12,0)}^{s w}, g(12,1)$ gives the highest social welfare. This proves part b.3) of the proposition.

(b.iii) For $0.9535<d<0.956154$, it follows that $k_{(12,12)-(12,1)}^{s w}<0<k_{(12,12)-(12,0)}^{s w}<$ $\bar{k}<k_{(12,1)-(12,0)}^{s w}$. Then, for either $k_{(12,12)-(12,1)}^{s w}<0 \leq k<k_{(12,12)-(12,0)}^{s w}<\bar{k}<$ $k_{(12,1)-(12,0)}^{s w}$ or $k_{(12,12)-(12,1)}^{s w}<0<k_{(12,12)-(12,0)}^{s w}<k<\bar{k}<k_{(12,1)-(12,0)}^{s w}$ the greatest social welfare is obtained with $g(12,1)$.

(b.iv) For $0.956154<d<1$, it follows that $k_{(12,12)-(12,1)}^{s w}<0<\bar{k}<k_{(12,12)-(12,0)}^{s w}<$ $k_{(12,1)-(12,0)}^{s w}$. Then, for all $0 \leq k<\bar{k}$ the greatest social welfare is obtained with $g(12,1)$.

\section{References}

[1] Belleflamme, P. and F. Bloch (2004). "Market Sharing Agreements and Collusive Networks," International Economic Review 45(2): 387-411.

[2] Bernheim, B.D. and M.D. Whinston (1998). "Exclusive Dealing," Journal of Political Economy 106: 64-103.

[3] Besanko, D. and M.K. Perry (1994). "Exclusive Dealing in a Spatial Model of Retail Competition," International Journal of Industrial Organization 12: 297-329.

[4] Betancourt, R.R. (2004), The Economics of Retailing and Distribution. London: Edward Elgar Publishing Ltd.

[5] Caballero-Sanz, F. and P. Rey (1996). "The Policy Implications of the Economic analysis of Vertical Restraints," Economic Papers-European Commission, DG for Economic and Financial Affairs, Number 119. 
[6] Calvó-Armengol, A. (2004). "Job Contact Networks," Journal of Economic Theory 115: 191-206.

[7] Calvó-Armengol, A. and M.O. Jackson (2004). "The Effects of Social Networks on Employment and Inequality," American Economic Review 94(3): 426-454.

[8] Chang, M.H. (1992). "Exclusive Dealing Contracts in a Successive Duopoly with Side Payments," Southern Economic Journal 59: 180-93.

[9] Dobson, P.W. and M. Waterson (1997). "Exclusive Trading Contracts in Successive Differentiated Duopoly," Southern Economic Journal 63: 361-77.

[10] Goyal, S. and S. Joshi (2003). "Networks of Collaboration in Oligopoly," Games and Economic Behavior 43(1): 57-85.

[11] Goyal, S. and J.L. Moraga-Gonzalez (2001). "R\&D Networks," Rand Journal of Economics 32(4): 686-707.

[12] Hendricks, K., M. Piccione and G. Tang (1997). "Entry and Exit in Hub-Spoke Networks," Rand Journal of Economics 28: 291-303.

[13] Iyer, G. and J.M. Villas-Boas (2003). "A Bargaining Theory of Distribution Channels," Journal of Marketing Research 40: 80-100.

[14] Jackson, M.O. (2003). "The Stability and Efficiency of Economic and Social Networks," in Networks and Groups: Models of Strategic Formation, edited by B. Dutta and M.O. Jackson. Heidelberg: Springer-verlag.

[15] Jackson, M.O. (2005). "A Survey of Models of Network Formation: Stability and Efficiency," in Group Formation in Economics: Networks, Clubs and Coalitions, edited by G. Demange and M. Wooders. Cambridge: Cambridge University Press.

[16] Jackson, M.O. and A. van den Nouweland (2005). "Strongly Stable Networks," forthcoming in Games and Economic Behavior.

[17] Jackson, M.O. and A. Wolinsky (1996). "A Strategic Model of Social and Economic Networks," Journal of Economic Theory 71: 44-74.

[18] Kranton, R. and D. Minehart (2000a). "Networks versus Vertical Integration," Rand Journal of Economics 31: 570-601.

[19] Kranton, R. and D. Minehart (2000b). "Competition for Goods in Buyer-Seller Networks," Review of Economic Design 5: 301-331. 
[20] Kranton, R. and D. Minehart (2001). "Theory of Buyer-Seller Networks," American Economic Review 91(3): 485-508.

[21] Lin, Y.J. (1990). "The Dampening-of-Competition Effect of Exclusive Dealing," Journal of Industrial Economics 39: 209-23.

[22] Mycielski, J., Y.E. Riyanto and F. Wuyts (2000)." Inter- and Intrabrand Competition and the Manufacturer-Retailer Relationship," Journal of Institutional and Theoretical Economics 156: 599-624.

[23] Moner-Colonques, R., J. J. Sempere-Monerris and A. Urbano (2004). "The Manufacturers' Choice of Distribution Policy under Successive Duopoly," Southern Economic Journal 70(3): 532-548.

[24] Nishiguchi, T. (1994), Strategic Industrial Sourcing: The Japanese Advantage. New York: Oxford University Press.

[25] O'Brien, D. and G. Shaffer (1993). "On the Dampening-of-Competition Effects of Exclusive Dealing," Journal of Industrial Economics 41: 215-221.

[26] O'Brien, D.P. and G. Shaffer (1997). "Nonlinear Supply Contracts, Exclusive Dealing and Equilibrium Market Foreclosure," Journal of Economics and Management Strategy 6: 755-85.

[27] Rey, P. and J. Stiglitz (1995). "The Role of Exclusive Territories in Producers' Competition," Rand Journal of Economics 26: 431-51.

[28] Rey, P. and J. Tirole (2003). "A Primer on Foreclosure," forthcoming in Handbook of Industrial Organization Vol.3, edited by M. Armstrong and R. Porter. Amsterdam: Elsevier Science Publishers.

[29] Sass, T.R. (2005). "The Competitive Effects of Exclusive Dealing: Evidence from the U.S. Beer Industry," forthcoming in International Journal of Industrial Organization.

[30] Slade, M.E.(1998). "Beer and the Tie: Did Divestiture of Brewer-Owned Public Houses Lead to Higher Beer Prices?," The Economic Journal 108: 565-602.

[31] Tirole, J. (1988). The Theory of Industrial Organization. Cambridge, MA: The MIT Press.

[32] Wang, P. and A. Watts (2003). "Formation of Buyer-Seller Trade Networks in a Quality-Differentiated Product Market," mimeo: Vanderbilt University and Southern Illinois University. 
Département des Sciences Économiques de l'Université catholique de Louvain

Institut de Recherches Économiques et Sociales

Place Montesquieu, 3

1348 Louvain-la-Neuve, Belgique 